\title{
Exploring the Evolution of Star Formation and Dwarf Galaxy Properties with JWST/MIRI Serendipitous Spectroscopic Surveys
}

\author{
Matteo Bonato ${ }^{1}$, Anna Sajina ${ }^{1}$, Gianfranco De Zotti ${ }^{2,3}$, Jed McKinney ${ }^{1}$, Ivano Baronchelli ${ }^{4}$, Mattia Negrello ${ }^{5}$, Danilo Marchesini ${ }^{1}$, \\ Eric Roebuck ${ }^{1}$, Heath Shipley ${ }^{1}$, Noah Kurinsky ${ }^{6}$, Alexandra Pope ${ }^{7}$, Alberto Noriega-Crespo ${ }^{8}$, Lin Yan ${ }^{4}$, and Allison Kirkpatrick ${ }^{9}$ \\ ${ }^{2}$ Department of Physics \& Astronomy, Tufts University, 574 Boston Avenue, Medford, MA, USA \\ ${ }^{2}$ INAF, Osservatorio Astronomico di Padova, Vicolo Osservatorio 5, I-35122 Padova, Italy \\ ${ }^{3}$ SISSA, Via Bonomea 265 , I-34136 Trieste, Italy \\ ${ }^{4}$ California Institute of Technology, Pasadena, CA, USA \\ ${ }^{5}$ School of Physics and Astronomy, Cardiff University, Queens Buildings, The Parade, Cardiff CF24 3AA, UK \\ ${ }^{6}$ Department of Physics, Stanford University, Stanford, CA, USA \\ ${ }^{7}$ Department of Astronomy, University of Massachusetts Amherst, Amherst, MA, USA \\ ${ }^{8}$ Space Telescope Science Institute, 3700 San Martin Drive, Baltimore, MD, USA \\ ${ }^{9}$ Department of Astronomy, Yale University, New Haven, CT, USA \\ Received 2016 August 1; revised 2017 January 16; accepted 2017 January 24; published 2017 February 17
}

\begin{abstract}
The James Webb Space Telescope's Medium Resolution Spectrometer (MRS), will offer nearly two orders of magnitude improvement in sensitivity and $>3 \times$ improvement in spectral resolution over our previous space-based mid-IR spectrometer, the Spitzer IRS. In this paper, we make predictions for spectroscopic pointed observations and serendipitous detections with the MRS. Specifically, pointed observations of Herschel sources require only a few minutes on source integration for detections of several star-forming and active galactic nucleus lines, out to $z=3$ and beyond. But the same data will also include tens of serendipitous $0 \lesssim z \lesssim 4$ galaxies per field with infrared luminosities ranging $\sim 10^{6}-10^{13} L_{\odot}$. In particular, for the first time and for free we will be able to explore the $L_{\mathrm{IR}}<10^{9} L_{\odot}$ regime out to $z \sim 3$. We estimate that with $\sim 100$ such fields, statistics of these detections will be sufficient to constrain the evolution of the low- $L$ end of the infrared luminosity function, and hence the star formation rate function. The above conclusions hold for a wide range in the potential low- $L$ end of the IR luminosity function, and account for the PAH deficit in low- $L$, low-metallicity galaxies.
\end{abstract}

Key words: galaxies: active - galaxies: evolution - galaxies: luminosity function, mass function galaxies: starburst - infrared: galaxies

\section{Introduction}

Over the last few decades it has become clear that the bulk of the galaxy star formation (SF) and supermassive black hole (SMBH) accretion in the universe occurred in the redshift interval $1 \lesssim z \lesssim 3$ (Merloni \& Heinz 2008; Delvecchio et al. 2014; Madau \& Dickinson 2014). The correlation between the mass of SMBHs located at the galaxy centers and the properties of the spheroidal stellar components (see Ferrarese \& Ford 2005; Kormendy \& Ho 2013, for reviews) reveal a connection between the black hole growth and the build-up of the mass in stars (see e.g., Hopkins et al. 2006, 2008). The debate about the nature of this connection (i.e., a direct interaction, an indirect connection, or a correlation arising from availability of free baryons) is open (see e.g., Peng 2007; Jahnke \& Macciò 2011; Graham \& Scott 2013). Moreover, the evolution of this connection with cosmic time is largely unknown. To shed light on this issue, a detailed investigation of the physical processes operating in large samples of cosmic sources is necessary. Moreover, the most active SF phases of galaxies and the associated active galactic nucleus (AGN) growth are severely dust-obscured (see e.g., Burgarella et al. 2013; Madau \& Dickinson 2014) and therefore best studied at IR wavelengths.

In particular, the mid-IR (MIR) regime (here defined as restframe $\sim 2-30 \mu \mathrm{m}$ ) is rich in spectral lines and features that provide excellent diagnostics of the power source (SF or AGN) as well as of the level of obscuration and dust composition (e.g., Sajina et al. 2007; Yan et al. 2007; Pope et al. 2008;
Dasyra et al. 2009; Sajina et al. 2009, 2012; Kirkpatrick et al. 2012, 2015). In this regime, fine-structure lines come from star-forming regions or from nuclear activity or from both. MIR line ratio diagrams can be used to identify composite sources and distinguish between emission from star-forming regions and emission excited by nuclear activity (Genzel et al. 1998; Lutz et al. 1999; Sturm et al. 2002; Armus et al. 2007; Farrah et al. 2007; Ho \& Keto 2007; Yuan et al. 2010). The PAH luminosities have been calibrated as star formation rate (SFR) indicators (e.g., Roussel et al. 2001, Förster Schreiber et al. 2004, Peeters et al. 2004, Desai et al. 2007; Shipley et al. 2016).

Thanks to its unprecedented sensitivity and resolution (Glasse et al. 2015), a giant leap in this field is expected from the James Webb Space Telescope (JWST ${ }^{10}$; Gardner et al. 2006, Windhorst et al. 2009, Finkelstein et al. 2015) Mid-Infrared Instrument (MIRI; Rieke et al. 2015). For example its Medium Resolution Spectrometer (MRS) will provide a gain in sensitivity of 1-2 orders of magnitudes over the Spitzer Infrared Spectrograph (Houck et al. 2004b) and $\gtrsim 3 \times$ gain in spectral resolution. This advancement will allow us to obtain, at high $\mathrm{z}$, the high- and medium-resolution spectra necessary for the detections of the fine-structure AGN lines, the study of which has mainly been limited to the local universe so far (e.g., Sturm et al. 2002; Armus et al. 2007; Farrah et al. 2007; Dasyra et al. 2011; Inami et al. 2013). Therefore, MRS-pointed observations will make it possible to not only obtain

${ }^{10}$ http://www.jwst.nasa.gov 
spectroscopic redshifts for essentially any known dusty galaxy population across the bulk of cosmic time, but also to accurately measure the contributions from stellar and AGN activity to their luminosity.

Current photometric surveys of dusty galaxies are biased toward higher luminosity sources because even in the local universe they are limited to $L_{\mathrm{IR}}>4 \times 10^{7} L_{\odot}$ galaxies and by $z \sim 2$ - the peak epoch for SF and black hole accretion activity - they are limited to $L_{\mathrm{IR}}>3 \times 10^{11} L_{\odot}$ galaxies (see e.g., Sanders et al. 2003; Eales et al. 2010; Magnelli et al. 2011; Clemens et al. 2013; Gruppioni et al. 2013, Lutz 2014). ${ }^{11}$ The minimum IR luminosities achieved by the deepest Spitzer MIR spectroscopic observations are $\sim 10^{9} L_{\odot}$ at $z \sim 0$ and $\sim 10^{11} L_{\odot}$ at $z \sim 2$ (see Sajina et al. 2008; Dasyra et al. 2009; O'Dowd et al. 2009; Shipley et al. 2013). The unprecedented sensitivity of the MRS will allow us to access IR luminosities about two orders of magnitude lower than current limits. The space density of such dwarf galaxies is high enough that we expect to be able to serendipitously detect them within the fields of any MRS-pointed observation. Therefore, not only will the JWST-MRS allow us to gain much greater insight into the physical conditions and power sources of known dusty galaxy populations across cosmic history, but it will also allow for the discovery of new populations in the hitherto largely unknown low- $L$, low-metallicity, high- $z$ regime.

Indeed, the $L_{\mathrm{IR}} \lesssim 10^{9} L_{\odot}$ luminosity regime is largely unexplored and poorly constrained by current galaxy evolution models. So far, IR continuum and PAH emission of dwarf starforming galaxies has been studied only in the Local Group and in other nearby sources (Gallagher et al. 1991; Israel et al. 1996; Contursi et al. 1998, 2000; Reach et al. 2000; Sturm et al. 2000; Hunter et al. 2001; Vermeij et al. 2002; Houck et al. 2004a; Engelbracht et al. 2005, 2008; Jackson et al. 2006; Madden et al. 2006, 2013; Galametz et al. 2009; Rémy-Ruyer et al. 2013; Fisher et al. 2014). These studies suggest that the SEDs of low-metallicity dwarf galaxies may be very different than typical star-forming galaxies (see e.g., Houck et al. 2004a). The density of these low- $L$ star-forming dwarf galaxies in the universe is unknown (see e.g., Lutz 2014). Additionally, the relationships between continuum and line luminosity in this luminosity range are very unsure. This is due to the fact that the physical conditions in such low- $L$ galaxies differ from those in their high- $L$ counterparts, which affects line excitation. Therefore a simple extrapolation (at low luminosities) of the line-to-continuum relations calibrated at high- $L$ could be deceiving. For example, the well-known massmetallicity relation (Tremonti et al. 2004; Zahid et al. 2013) indicates that the metallicity decreases at lower masses (and therefore at lower luminosities). This effect should be stronger at higher redshifts, because metallicity decreases with increasing $z$ (Zahid et al. 2013). MRS detections (as well as non-

\footnotetext{
11 In Sanders et al. (2003), the minimum $L_{\mathrm{IR}}$ in the Revised Bright Galaxy Sample (mean redshift of the sample $z \sim 0.01$ ), observed by the Infrared Astronomical Satellite (96 percent of the sky) at 12, 25, 60, and $100 \mu \mathrm{m}$ is $\sim 4 \times 10^{7} L_{\odot}\left(\right.$ only three galaxies of the sample have $\left.L_{\mathrm{IR}}<10^{8} L_{\odot}\right)$. At $z \sim 2$, Magnelli et al. (2011; in the redshift bin $1.8 \lesssim z \lesssim 2.3$ ) and Gruppioni et al. (2013; $1.7 \lesssim z \lesssim 2.0$ ) achieve a minimum IR luminosity of $\sim 3 \times 10^{11} L_{\odot}$. Magnelli et al. (2011) used Spitzer MIPS $(24,70 \mu \mathrm{m})$ and IRAC $(3.6 \mu \mathrm{m})$ observations of the GOODS North and South fields (area $\sim 100 \operatorname{arcmin}^{2}$ ). Gruppioni et al. (2013) used Herschel PACS observations (at 70, 100, and $160 \mu \mathrm{m}$ ) in combination with Herschel SPIRE data (at 250, 350, and $500 \mu \mathrm{m}$ ), over the GOODS North and South, Extended Chandra Deep Field South and Cosmic Evolution Survey areas (total area $\sim 2.4 \mathrm{deg}^{2}$ ).
}

detections) of these low- $L$ galaxies will allow us to explore the physical properties of such sources.

In this paper, we look at the star-forming and AGN line detectability for MIRI MRS-pointed observations. We present predictions for the number, luminosity, and redshift distribution of serendipitously detected galaxies and AGN within the fields of the same pointed observations. We make our predictions based on three different assumptions about the unknown low- $L$ end of the IR luminosity function, and accounting for the potential PAH deficit in low- $L$, low-metallicity galaxies.

The paper is organized as follows. In Section 2, we present the key technical aspects of the MRS relevant to this paper. In Section 3, we summarize our modeling approach. In Section 4, we present predictions for MRS-pointed observations and serendipitous surveys. In Section 5, we discuss the implications of our results for the study of the galaxy-AGN (co-)evolution and of the low-luminosity galaxy properties. Section 6, contains a summary of our main conclusions. Throughout this paper we adopt a flat $\Lambda \mathrm{CDM}$ cosmology with matter density $\Omega_{\mathrm{m}} \sim 0.31$, dark energy density $\Omega_{\Lambda} \sim 0.69$, and Hubble constant $h=H_{0} / 100 \mathrm{~km} \mathrm{~s}^{-1} \mathrm{Mpc}^{-1} \sim 0.67 \quad$ (Planck Collaboration 2015).

\section{MRS Overview}

The MIRI MRS instrument (Wells et al. 2015) covers wavelengths from $\sim 4.9$ to $\sim 28.8 \mu \mathrm{m},{ }^{12}$ with $R \sim 2200-3500$ and a Field of View (FoV) from $\sim 3$ ! $0 \times 3$ !! 9 to $\sim 6$ ! $7 \times$ 7!.7. ${ }^{13}$ It consists of four Integral Field Units working simultaneously (Gordon et al. 2015). Consistent with Glasse et al. (2015), the reference extended source detection limits ${ }^{12}$ $(10 \sigma, 10,000 \mathrm{~s})$ for the four channels are $1.8 \times 10^{-20} \mathrm{~W} \mathrm{~m}^{-2} /$

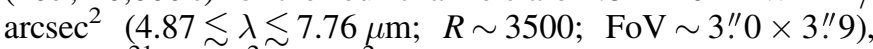
$9.0 \times 10^{-21} \mathrm{~W} \mathrm{~m}^{-2} / \operatorname{arcsec}^{2} \quad(7.45 \lesssim \lambda \lesssim 11.87 \mu \mathrm{m} ; \quad R \sim 2800 ;$ FoV $\sim 3$ ". $5 \times 4$ ". 4$), 5.0 \times 10^{-21} \mathrm{~W} \mathrm{~m}^{-2} / \operatorname{arcsec}^{2} \quad(11.47 \lesssim \lambda \lesssim$ $18.24 \mu \mathrm{m} ; \quad R \sim 2700 ; \quad$ FoV $\sim 5$ !" $2 \times 6$ ". 2$)$ and $1.9 \times$ $10^{-20} \mathrm{~W} \mathrm{~m}^{-2} / \operatorname{arcsec}^{2} \quad(17.54 \lesssim \lambda \lesssim 28.82 \mu \mathrm{m} ; \quad R \sim 2200 ;$ $\mathrm{FoV} \sim 6$ !" $7 \times 7 !$ ! $)$.

The spatial resolution varies from $\sim 0.2 \operatorname{arcsec}$ at $4.9 \mu \mathrm{m}$ to $\sim 1.1$ arcsec at $28.8 \mu \mathrm{m}$. Serendipitous detections within the fields of the pointed observations are operationally supported only for the MRS, ${ }^{14}$ not for the Low-Resolution Spectrometer (Kendrew et al. 2015).

\section{Modeling Approach}

\subsection{The Evolution of the IR Luminosity Function}

Our reference model for the evolution of the IR luminosity function is the model used in Kurinsky et al. (2017). The functional form for the luminosity function is a double powerlaw (e.g., Negrello et al. 2013), $\Phi_{P L}=\Phi^{*}\left[\left(\frac{L}{L^{*}}\right)^{\alpha}+\left(\frac{L}{L^{*}}\right)^{\beta}\right]^{-1}$; where $\Phi^{*}(z)=\Phi_{0}^{*}(1+z)^{p}$ and $L^{*}(z)=L_{0}^{*}(1+z)^{q} \cdot p$ denotes density evolution and $q$ denotes luminosity evolution;

\footnotetext{
12 The full $4.9-28.8 \mu \mathrm{m}$ wavelength coverage needs three different grating angle settings. This fact must be taken into account in the computation of the overheads, because the full spectra can be obtained in a time $3 \times$ the on-target integration time used throughout this paper.

13 http://ircamera.as.arizona.edu/MIRI/performance.htm

14 Hereafter, by number of serendipitous detections "per FoV" we mean the total number of detections (at $5 \sigma$ level) per single exposure, inside the different (superimposed) FoVs of the four MRS channels. The maximum area of sky covered in a single exposure is given by the FoV of the highest-wavelength channel (see Wells et al. 2015).
} 


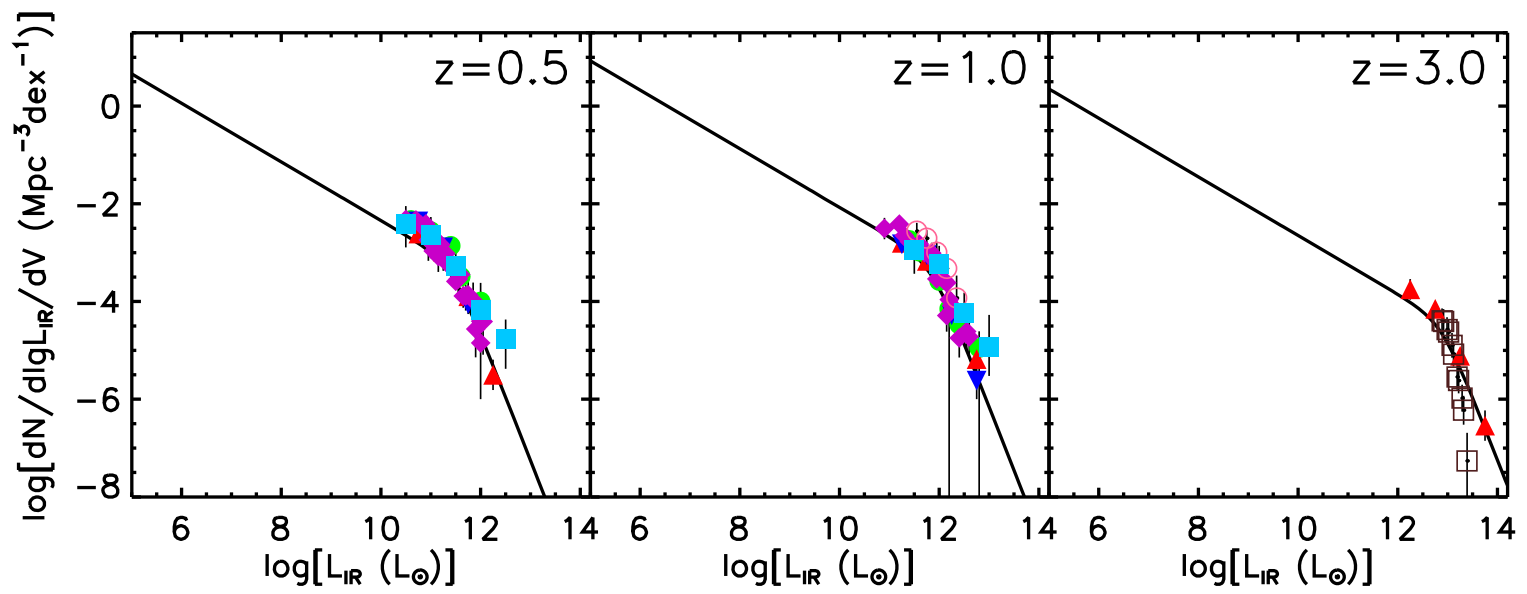

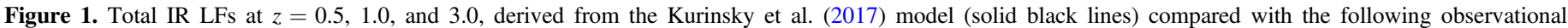

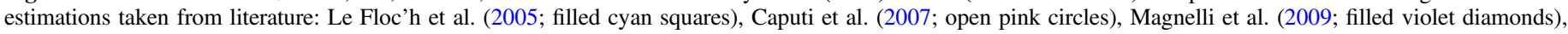

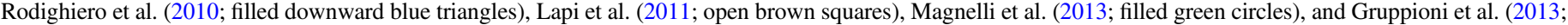
filled upward red triangles).

at two break redshifts the power of the density $\left(z_{b p}\right)$ and luminosity $\left(z_{b q}\right)$ evolution changes. The evolution of the AGN fraction is given by a power-law of the form: $f_{\mathrm{AGN}, z}=f_{\mathrm{AGN}, 0} *$ $\left[\log \left(L_{\mathrm{TIR}}\right) / 12\right]^{12} *(1+z)^{t}$; where $f_{\mathrm{AGN}, 0}$ represents the $z \sim 0$ AGN fraction at $\log \left(L_{\mathrm{TIR}} / L_{\odot}\right)=12 . t \equiv t_{1}$ until a break redshift, $z_{b t}$, and $t \equiv t_{2}$ at higher $\mathrm{z}$. The fraction of composite sources with respect to all AGN is given by a single parameter: $f_{\text {comp. }}$ The adopted best-fit values of the parameters (taken from Kurinsky et al. 2017) are as follows: $\log \left(L_{0}^{*} / L_{\odot}\right)=10.86_{-0.07}^{+0.06}$; $\log \left(\Phi_{0}^{*}\right)=-3.24_{-0.05}^{+0.06} ; \quad z_{b p}=1.66_{-0.00}^{+1.43} ; \quad z_{b q}=1.85_{-0.29}^{+1.20} ; \quad p_{1}=$ $0.16_{-1.53}^{+1.54} ; q_{1}=3.33_{-0.86}^{+0.89} ; p_{2}=-3.32_{-1.91}^{+2.33} ; f_{\mathrm{AGN}, 0}=0.26_{-0.10}^{+0.13}$; $t_{1}=-0.14_{-0.67}^{+0.87} ; \quad t_{2}=0.12_{-3.25}^{+4.38} ; \quad z_{b t}=2.60_{-0.44}^{+0.63} ; \quad f_{\text {comp }}=$ $0.33_{-0.10}^{+0.43}$. In such a model, the slope parameters are set as $\alpha=2.6$ and $\beta=0.6$. These best-fit parameters are found by fitting HerMES-COSMOS MIPS/SPIRE color-magnitude diagrams. This data set yields strong constraints on the evolution of the IR LF up to $z \sim 1.5$. On the other hand, it is not deep enough to effectively constrain the model at higher redshifts. We are working on extending these constraints to higher redshifts by applying the code to deeper samples (M. Bonato et al. 2017, in preparation). For the purposes of this paper, we have also required consistency of the model with observational determinations of the IR LF at higher $z$. Observational estimates of the IR LF are currently available up to $z \simeq 4$ (Gruppioni et al. 2013). This required the modification of only one parameter, $q_{2}$. We adopt $q_{2}=1.6$ to fit literature LF determinations at $z \gtrsim 2$. Figure 1 compares our reference model LF at three representative redshifts $(0.5,1$, and 3$)$ with the available data.

The adopted SED library, based on Kirkpatrick et al. (2015), includes templates for star-forming galaxies, composites, and AGN, as well as their redshift and luminosity evolution. It is based on a sample of 343 dusty galaxies with mid-IR IRS spectra and a wealth of ancillary data covering the near- to farIR regime. In particular, the IRS spectra allow for accurate spectral classification into the three different SED types. This library does not include a low-metallicity starburst template.

In our reference model, as in all existing IR luminosity function models, the low- $L$ regime is pure extrapolation. Even in the local universe we have constraints on the IR LF only essentially above $\sim 10^{8} L_{\odot}$ (see, e.g., Sanders et al. 2003; Clemens et al. 2013; Lutz 2014). Indeed, assuming a constant, relatively steep, $<L_{*}$ slope quickly reaches unrealistically large space densities: the maximum values found by deep optical surveys are $\sim 0.1 \mathrm{Mpc}^{-3} \mathrm{dex}^{-1}$ (e.g., Driver et al. 2012). Generally, we expect a break of the IR LFs at low luminosities for a simple physical reason: very small galaxies have a low binding energy and therefore cannot bear substantial SFR because the mechanical energy released by only a few supernovae is sufficient to unbind the gas, thus halting the SF. At low-z, low-IR luminosities (typically below $\sim 10^{9}-$ $10^{10} L_{\odot}$ ) may be due to dust heated by the general radiation field produced by older stellar populations, rather than newly born stars. Obviously in this case we do not expect lines excited by SF. Note also that a fraction of low-IR luminosity objects are massive early-type galaxies, with high bolometric luminosity but almost devoid of gas and dust and consequently with very low SFR.

To account for the above uncertainties, we test different assumptions about the low-luminosity end of the IR LF. Specifically, we test three cases (shown in Figure 2 for a reference redshift of $z=0.5$ ): (1) extrapolate the current model down to $10^{5} L_{\odot}$ (upper limit); (2) level off the faint end of the IR LF at a density of $0.1 \mathrm{Mpc}^{-3} \mathrm{dex}^{-1}$ (intermediate case); (3) impose a low-luminosity cut-off at all $z$ at the luminosity corresponding to a density of $0.1 \mathrm{Mpc}^{-3} \mathrm{dex}^{-1}$ (lower limit). The last case is for the extreme scenario where at low- $L$ we do not expect any SF or AGN lines (what we ultimately detect with the MRS) because 100 percent of the luminosity is due to old stellar populations. These three cases are all compatible with the current constraints given by the observational determinations of the Cosmic Infrared Background (CIB) intensity. In fact the contribution of these very low- $L$ sources to the total CIB is minor, and the variation is only a few percents among the three cases. In this paper the "intermediate case" for the Kurinsky et al. (2017) model is adopted as reference model, but we compare the results with those obtained using the two extreme cases on either side of the reference model (Case 1 and Case 3).

\subsection{Galaxy Sizes}

Because of the very small FWHM, a significant fraction of the galaxies will be spatially resolved by the JWST. To estimate the galaxy sizes in our simulations (depending mainly on 


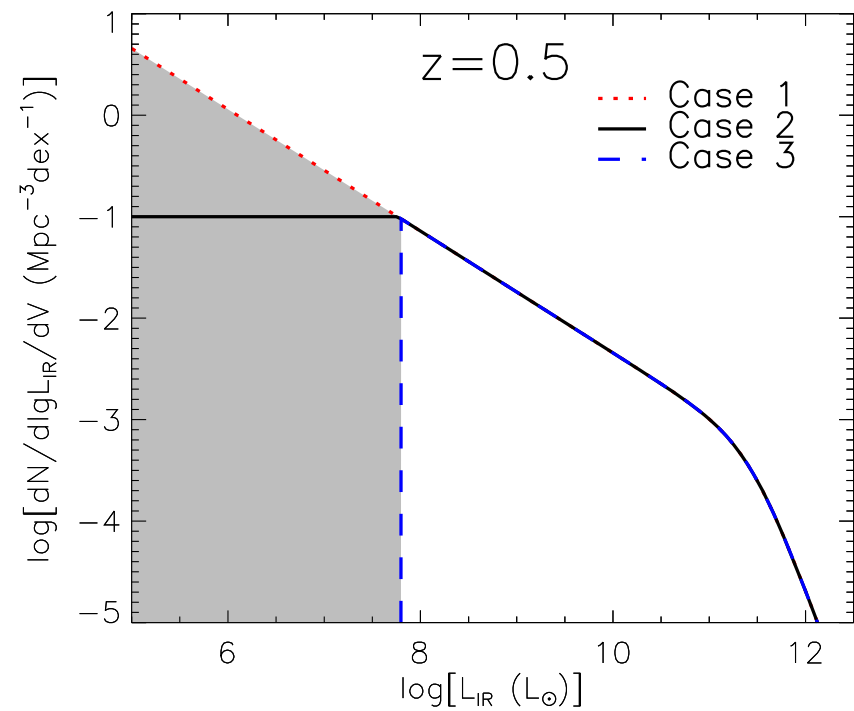

Figure 2. At $z=0.5$ the total IR LF of the Kurinsky et al. (2017) model with three different assumptions for the low $L$ end (shaded region): case $1-$ extrapolate current models down to $10^{5} L_{\odot}$ (dotted red line); case 2-break such that all low- $L$ galaxies have a density of $0.1 \mathrm{Mpc}^{-3} \mathrm{dex}^{-1}$ (solid black line); case 3-growth until a density of $0.1 \mathrm{Mpc}^{-3} \mathrm{dex}^{-1}$ and null after that (dashed blue line).

luminosity and stellar mass), we derived their SFR using the $L_{\mathrm{IR}} / \mathrm{SFR}$ relation by Clemens et al. (2013). The SFR was converted into stellar mass using the main sequence empirical correlations of star-forming galaxies obtained by Kurczynski et al. (2016) in the redshift range $0.5 \lesssim z \lesssim 3.0$. For $z<0.5$, we used the Peng et al. (2010) correlation, measured at $z \sim 0.2$; at higher $z$, the relations (at $z \sim 4,5$, and 6) are derived by Salmon et al. (2015). Finally the stellar mass content was converted into an effective radius through the Shen et al. (2003) best-fit relation ${ }^{15}$ at $z<1$ and through the relation estimated by Bruce et al. (2012) ${ }^{16}$ at higher redshift. For example, for a galaxy having $L_{\mathrm{IR}} \sim 10^{12} L_{\odot}$ we obtain an effective radius of $\sim 1$ !' 13 (at $z \sim 0.5$; corresponding to $\sim 6.6 \mathrm{kpc}$ ) and $\sim 0$ !. 14 (at $z \sim 2.0 ; \sim 1.1 \mathrm{kpc}$ ).

We caution that the sizes associated with some simulated galaxies in our analysis are probably overestimated. Specifically, the SFR $/ M_{*}$ ratio for galaxies that are starbursting could be significantly higher than the main sequence relations used here (see, e.g., Rodighiero et al. 2014). Moreover, there is evidence that the sizes of galaxies in the MIR may be smaller than in the optical regime (see, e.g., Díaz-Santos et al. 2010; Xu et al. 2014). Given that more compact galaxies of the same luminosity are easier to detect than more extended ones, this potential overestimation of the sizes means that our results can be viewed as a conservative lower limit on the numbers of serendipitous detections.

\subsection{Correlations Between Continuum and Line Luminosity}

To estimate the counts of galaxy and AGN line detections by MIRI surveys, we coupled the redshift-dependent IR luminosity functions of the source populations with relationships between line and continuum luminosities.

\footnotetext{
15 Their Equation (18), for late-type galaxies, with parameter values listed in the last line of their Table 1; we considered a range of effective radii $(1-10 \mathrm{Kpc})$ consistent with their fitting (see their Figure 11).

${ }^{16}$ For their sample of $1<z<3$ massive galaxies, they find a median size of a factor $\sim 2.3$ times smaller than comparably massive local galaxies.
}

We used the calibrations derived by Bonato et al. (2014a, 2014b, 2015) for 33 MIR fine-structure lines (placed in the rest-frame wavelength range $3.03 \lesssim \lambda \lesssim 25.98 \mu \mathrm{m}) ; 4$ PAH lines at 6.2, 7.7, 8.6 and $11.3 \mu \mathrm{m}$; and 2 silicate bands (in emission and in absorption) at 9.7 and $18.0 \mu \mathrm{m}$.

The 33 MIR fine-structure lines are the following:

1. Three coronal region lines: [Mg VIII] 3.03, [Si IX] 3.92 and [Si VII] $6.50 \mu \mathrm{m}$;

2. Thirteen AGN fine-structure emission lines: [Ca IV] 3.21, $[\mathrm{Ca} \mathrm{V}] 4.20,[\mathrm{Mg} \mathrm{IV}] 4.49, \quad[\mathrm{Ar} \mathrm{VI}]$ 4.52, [Mg V] 5.60, $[\mathrm{Ne} V \mathrm{VI}] 7.65,[\mathrm{Ar} \mathrm{V}] 7.90,[\mathrm{Ca} \mathrm{V}]$ 11.48, [Ar V] 13.09, $[\mathrm{Mg} \mathrm{V}] 13.50, \quad[\mathrm{Ne} \mathrm{V}] 14.32, \quad[\mathrm{Ne} \mathrm{V}] 24.31$ and [O IV] $25.89 \mu \mathrm{m}$;

3. Nine stellar/H II region lines: [Ar II] 6.98, [Ar III] 8.99, [S IV] 10.49, H I 12.37, [Ne II] 12.81, [Cl II] 14.38, [Ne III] 15.55, [S III] 18.71 and [Ar III] $21.82 \mu \mathrm{m}$;

4. Three lines from photodissociation regions: [Fe II] 17.93, [Fe III] 22.90 and [Fe II] $25.98 \mu \mathrm{m}$;

5. Five molecular hydrogen lines: $\mathrm{H}_{2}$ 5.51, $\mathrm{H}_{2} 6.91, \mathrm{H}_{2} 9.66$, $\mathrm{H}_{2} 12.28$ and $\mathrm{H}_{2} 17.03 \mu \mathrm{m}$.

For the PAH 6.2, РAH 7.7, РAH 8.6, PAH 11.3, Sil9.7, Sil18.0, $\mathrm{H}_{2}$ 9.66, [S IV]10.49, $\mathrm{H}_{2}$ 12.28, [Ne II]12.81, [Ne V] 14.32, [Ne III]15.55, $\mathrm{H}_{2}$ 17.03, [S III]18.71, [Ne V]24.31, and [O IV] $25.89 \mu \mathrm{m}$ lines, we used the relationships derived by Bonato et al. (2014a, 2014b, 2015) on the basis of observations collected from the literature. For all the other lines with either insufficient or missing data, the line-to-continuum luminosity relations were derived in Bonato et al. (2015) using the IDL Tool for Emission-line Ratio Analysis (ITERA) written by Brent Groves. ${ }^{17}$ ITERA makes use of the library of published photoionization and shock models for line emission of astrophysical plasmas produced by the Modelling And Prediction in PhotoIonised Nebulae and Gasdynamical Shocks (MAPPINGS III) code. Among the options offered by ITERA in Bonato et al. (2015), we chose the Dopita et al. (2006) models for starbursts and, for AGNs, the dust free isochoric narrow-line region (NLR) models for type 1 and the dusty radiation-pressure dominated NLR models for type 2 (Groves et al. 2004). The chosen models were those that provided the best overall fit (minimum $\chi^{2}$ ) to the observed line ratios of local starbursts in the Bernard-Salas et al. (2009) catalog and of AGNs in the sample built by Bonato et al. (2014b) combining sources from the Sturm et al. (2002), Tommasin et al. (2008, 2010), and Veilleux et al. (2009) catalogs. In Bonato et al. (2015), our theoretical calibrations were compared with observational data, showing a very good agreement and so proving the goodness of our procedure.

To this initial sample, we added the PAH bands at 3.3 and $12.7 \mu \mathrm{m}$ and the $\mathrm{Pa} \alpha$ (H I $1.88 \mu \mathrm{m})$ line.

For the PAH $3.3 \mu \mathrm{m}$ line, we collected data (on line and continuum luminosity) of star-forming galaxies from Rodríguez-Ardila \& Viegas (2003), Imanishi et al. (2008, 2010), Sajina et al. (2009), Lee et al. (2012), Kim et al. (2012), and Yamada et al. (2013). We excluded objects for which there was evidence for a substantial AGN contribution to the total IR luminosity. The left panel of Figure 3 shows the $3.3 \mu \mathrm{m}$ PAH line luminosities versus $L_{\mathrm{IR}}$ for both local and high- $z(z \sim 2)$ sources.

${ }^{17}$ http://ascl.net/1307.012 

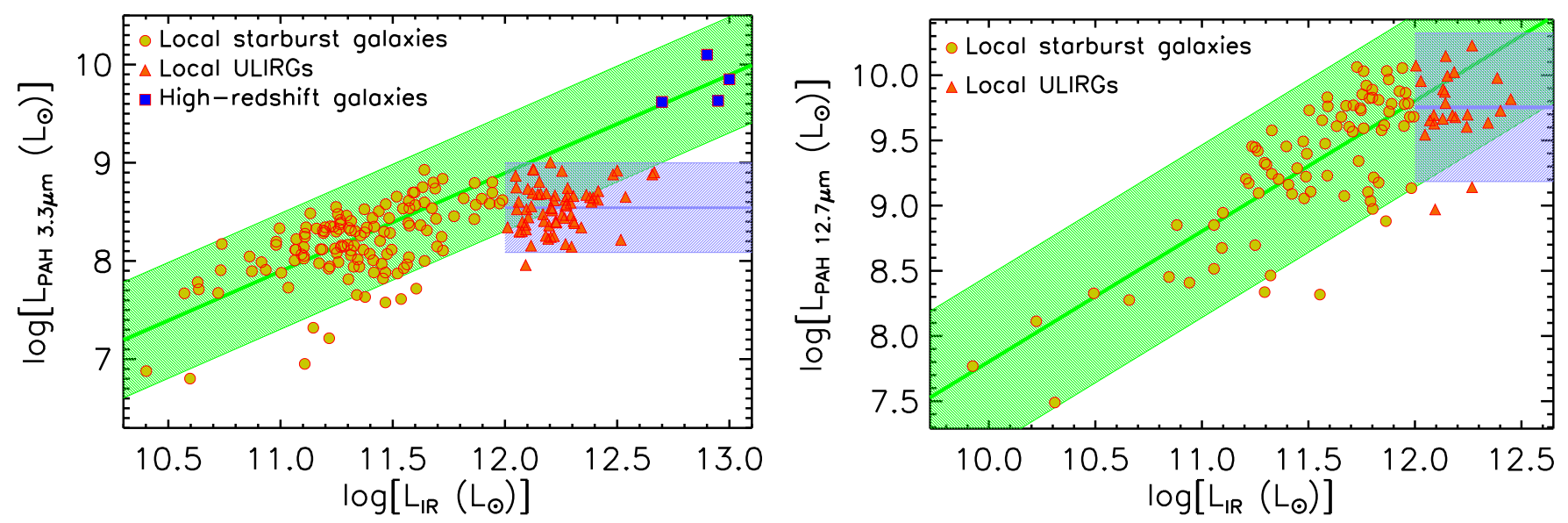

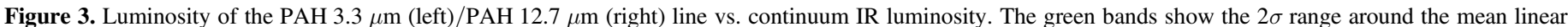

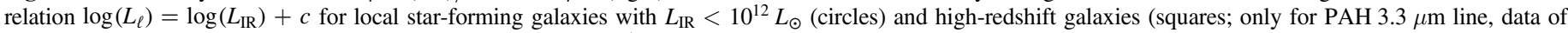

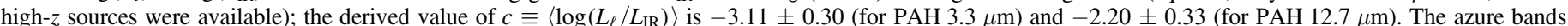

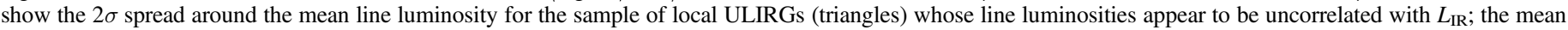
line luminosity $\left\langle\log L_{\ell}\right\rangle$ of these objects is $8.54 \pm 0.23$ (for PAH $3.3 \mu \mathrm{m}$ ) and $9.75 \pm 0.28$ (for PAH $12.7 \mu \mathrm{m}$ ).

Excluding local ULIRGS $\left(L_{\mathrm{IR}}>10^{12} L_{\odot}\right.$; orange triangles), which are known to be under-luminous in PAH lines (e.g., Bonato et al. 2014a and references therein), sources show a highly significant linear correlation, ${ }^{18}$ with mean $\left\langle\log \left(L_{\ell} / L_{\mathrm{IR}}\right\rangle=-3.11 \pm 0.30\right.$ where the "error," $\sigma=0.30$, is the dispersion around the mean. The correlation coefficient is $\simeq 0.8$, corresponding to a probability of no correlation of $\simeq 10^{-127}$.

More than 50 percent of the local ULIRGs lie on the right of the green band representing the $\pm 2 \sigma$ interval around the mean $\log \left(L_{\mathrm{IR}}\right)-\log \left(L_{\mathrm{PAH}}\right)$ relation holding for the other sources. Because local ULIRGs do not show any significant correlation between $\log \left(L_{\mathrm{IR}}\right)$ and $\log \left(L_{\mathrm{PAH}}\right)$, perhaps due to their limited luminosity range, we adopt a Gaussian distribution of the $\operatorname{logarithm}$ of the line luminosity, $\log \left(L_{\ell}\right)$, around a mean value, $\left\langle\log \left(L_{\ell}\right)\right\rangle=8.54$ with a dispersion of 0.23 . Actually assigning PAH luminosities to local ULIRGs using either the correlation or the Gaussian distribution does not significantly affect our results: in both cases all their PAH lines are detected by pointed observations with the minimum integration time considered here (see Section 5.2), and they give a negligible contribution to the counts of serendipitously detected sources, as expected given their very low space density. We checked that this conclusion holds independently of the transition redshift between "local" and "high- $z$ " ULIRGs by considering two extreme cases with transition redshifts of $z=0.2$ and $z=1.5$.

For the PAH $12.7 \mu \mathrm{m}$, we collected data from local starforming galaxies from Shipley et al. (2013) and Dasyra et al. (2009), which are shown on the right panel of Figure 3. Also in this case, we excluded objects for which there is evidence for a substantial AGN contribution to the total IR luminosity. As with the other PAH lines, we first investigated the correlation between line and IR luminosities excluding local ULIRGs. Again the data are consistent with a linear relation; we find

\footnotetext{
18 In Bonato et al. (2014a), we considered star-forming (stellar/H II and photodissociation region) lines and purely star-forming (non ULIRG) galaxies, exploiting the relationship between line and continuum luminosity with the support of extensive simulations (taking into account dust obscuration) and through the comparison with observational data. We showed that a direct proportionality between line and continuum luminosity (i.e., $\left.\log \left(L_{\ell}\right)=\log \left(L_{\mathrm{IR}}\right)+c\right)$ is preferable to a linear proportionality with a free slope (i.e., $\log \left(L_{\ell}\right)=a \times \log \left(L_{\mathrm{IR}}\right)+b$ ).
}

$\left\langle\log \left(L_{\ell} / L_{\mathrm{IR}}\right\rangle=-2.20 \pm 0.33\right.$. Although in this case local ULIRGs lie within the green band, for homogeneity with the treatment of the other PAH lines we adopt a Gaussian distribution with $\left\langle\log \left(L_{\ell}\right)\right\rangle=9.75$ and dispersion of 0.28 .

Apart from local ULIRGs, there are indications in the literature of a PAH emission deficit in low-metallicity starforming dwarf galaxies as well, compared with higher metallicity and/or higher luminosity star-forming galaxies (see e.g., Engelbracht et al. 2005, 2008; Madden et al. 2006; O'Halloran et al. 2006; Wu et al. 2006; Smith et al. 2007; Rosenberg et al. 2008; Galametz et al. 2009). Most of these studies suggest a decrement by a factor $\sim 10$ in the $L_{\mathrm{PAH}} / \mathrm{SFR}$ ratio, probably due to the fact that low-metallicity dwarf galaxies have very small grains and/or more hot dust. In particular, the lower PAH emission could be caused by the ability of the interstellar radiation field to penetrate lower dust column densities (lower dust-to-gas ratios are expected in lowmetallicity conditions), dissociating or destroying the PAHs (Galliano et al. 2003). We tested the consistency of our results considering the case of a deficit equal to 10 in PAH luminosity in all our simulated galaxies where $L_{\mathrm{IR}} \leqslant 10^{9} L_{\odot}{ }^{19}$ (at all $z$; see Section 4.2).

For the $P a \alpha$ line, we adopted the same procedure for theoretical calibrations described previously because of the scarcity of observational data useful for an empirical calibration. For star-forming galaxies, we obtained $\left\langle\log \left(L_{\ell} / L_{\mathrm{IR}}\right\rangle=\right.$ $-3.32 \pm 0.30$. This value is consistent, for example, with the relation adopted by Rieke et al. (2009; i.e., $\log \left(L_{P a \alpha} / L_{\mathrm{IR}}\right) \sim$ -3.14), who used the Kennicutt (1998) SFR $-L_{\mathrm{IR}}$ relation and a $\mathrm{Pa} \alpha / \mathrm{H \alpha}$ ratio of 0.128 (Hummer \& Storey 1987). We derived, as coefficients of the best-fit linear relations between line and AGN bolometric luminosities, $\log \left(L_{\ell}\right)=a$. $\log \left(L_{\mathrm{bol}}\right)+b$, and $1 \sigma$ dispersions associated to the relation: $a=0.85, b=-2.21$, disp $=0.34$.

We verified that our calibrations between line and continuum luminosity were consistent with the SED library adopted in Kurinsky et al. (2017). In particular, for the six PAH

\footnotetext{
${ }^{19}$ In Bonato et al. (2014a), our Figure 5, we showed the decrement of the linecontinuum luminosity ratio, at $L_{\mathrm{IR}} \leqslant 10^{9} L_{\odot}$, for several metal lines, probably due to metallicity. Similar results can be obtained from the mass-metallicity relation (see, e.g., Tremonti et al. 2004; Zahid et al. 2013).
} 

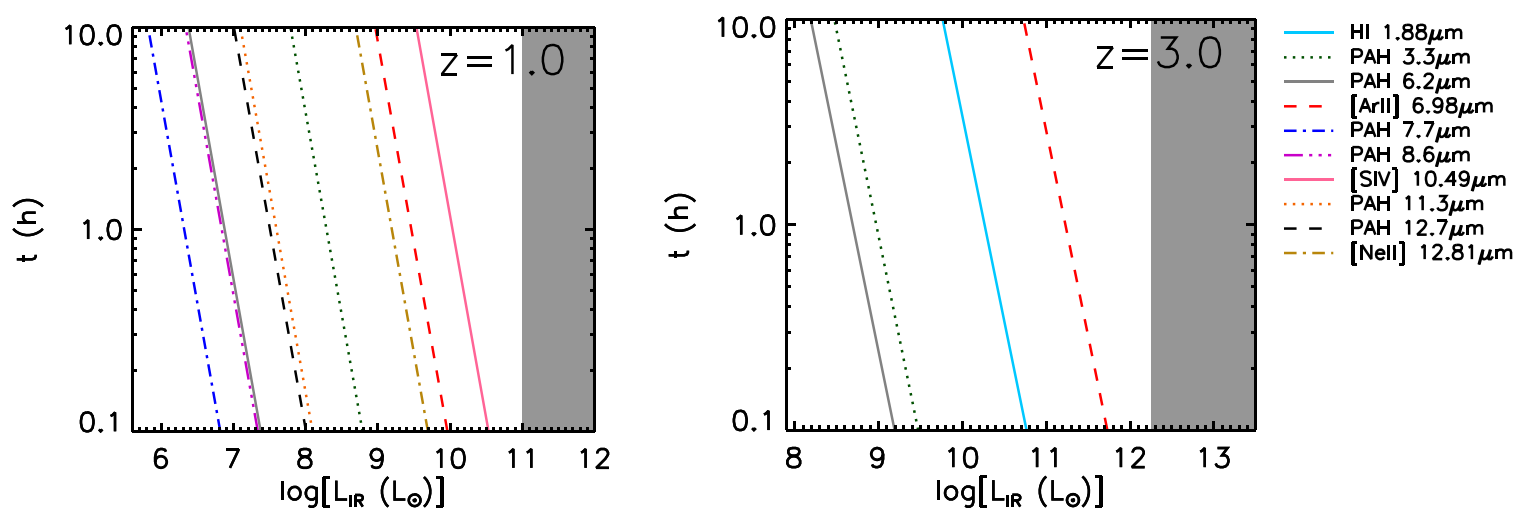

Figure 4. MIRI MRS exposure time vs. minimum IR luminosity (of star-forming galaxies) for a given line to be detected at $5 \sigma$. We show the predictions for sources at $z=1$ (left) and $z=3$ (right). In both cases, the shaded regions indicate the regime of Herschel/PACS and SPIRE-detected sources (based on Gruppioni et al. 2013). All the lines to the left of the shaded regions will be detected within the minimum time shown (i.e., $0.1 \mathrm{hr}$ ) in the Herschel sources. Only the key star-forming lines are shown for clarity. Almost all the omitted spectral lines $\left(\mathrm{H}_{2} 5.51, \mathrm{H}_{2} 6.91\right.$, [Ar III] 8.99, $\mathrm{H}_{2}$ 9.66, $\mathrm{H}_{2}$ 12.28, $\mathrm{H} \mathrm{I} 12.37,[\mathrm{Cl}$ II] 14.38, and Sil 9.7 $\mu$ m) are fainter than the lines shown in the figure.
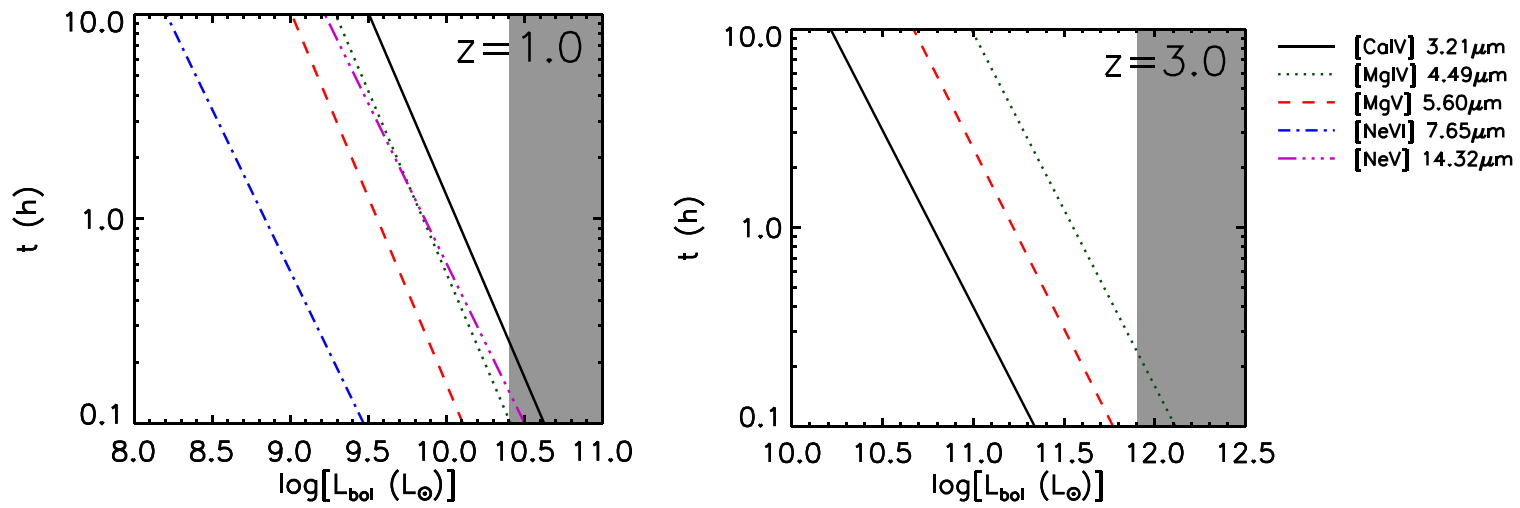

Figure 5. MIRI MRS exposure time vs. minimum bolometric luminosity (of AGN) for a given typical AGN line to be detected at $5 \sigma$. We show the predictions for sources at $z=1$ (left) and $z=3$ (right). In both cases, the shaded regions indicate the regime of Herschel/PACS- and SPIRE-detected AGN sources (based on Delvecchio et al. 2014). All the lines to the left of the shaded regions are detected within the minimum time shown (i.e., $0.1 \mathrm{hr}$ ) in the Herschel sources. Only the key AGN lines are shown for clarity. The omitted AGN lines ([Mg VIII] 3.03, [Si IX] 3.92, [Ca V] 4.20, [Ar VI] 4.52, [Si VII] 6.50, [Ar V] 7.90, [Ca V] 11.48, [Ar V] 13.09, and $[\mathrm{Mg} \mathrm{V}] 13.50 \mu \mathrm{m})$ are fainter than the lines shown in the figure.

features included in our line sample, we compared the $c=$ $\left\langle\log \left(L_{\ell} / L_{\mathrm{IR}}\right)\right\rangle$ of our calibrations with the mean values derived from the star-forming galaxy templates adopted in Kurinsky et al. (2017, taken from Rieke et al. 2009 and Kirkpatrick et al. 2015). From these SEDs, using the IDL tool PAHFIT (Smith et al. 2007), we obtained the following mean quantities (compared in parenthesis with the 1- $\sigma$ lower and upper limits of our calibrations): $c_{\mathrm{PAH} 3.3 \mu \mathrm{m}}=-3.12 \pm 0.54([-3.41,-2.81])$; $c_{\mathrm{PAH} 6.2 \mu \mathrm{m}}=-2.10 \pm 0.12([-2.56,-1.84]) ; c_{\mathrm{PAH} 7.7 \mu \mathrm{m}}=$ $-1.43 \pm 0.12([-2.00,-1.28]) ; c_{\mathrm{PAH}} 8.6 \mu \mathrm{m}=-2.20 \pm 0.14$ $([-2.52,-1.80]) ; c_{\mathrm{PAH}} 11.3 \mu \mathrm{m}=-2.07 \pm 0.20([-2.65,-1.93]) ;$ and $c_{\mathrm{PAH}} 12.7 \mathrm{~mm}=-2.31 \pm 0.11([-2.53,-1.87])$.

Aside from the PAH lines, all the other line calibrations are based on local observations. In this paper, we assume that these relations are not subject to redshift evolution. Indeed, MIRI will be the first instrument that will be able to test this assumption explicitly.

The line luminosity functions of all the lines of our sample have been computed starting from the redshift-dependent IR luminosity functions given by the evolutionary models. To properly take into account the dispersion in the relationships between line and continuum luminosities, we used the Monte Carlo approach described in Bonato et al. (2014a).

\section{MIRI MRS Predictions}

\subsection{Pointed Observations}

It is likely that some of the earliest pointed observations with the MRS will focus on the IR-luminous dust-obscured populations, such as the Herschel-selected galaxies (e.g., Eales et al. 2010; Elbaz et al. 2011; Oliver et al. 2012; Gruppioni et al. 2013; Bourne et al. 2016; Valiante et al. 2016). Figures 4 and 5 show the MRS exposure times needed to achieve a $5 \sigma$ detection of SF and AGN lines at $z=1$ and $z=3$ as a function of the IR luminosity (of the SF component) and of the bolometric luminosity (of the AGN component), respectively. ${ }^{20}$ The shaded areas correspond to the IR luminosities represented in the SF luminosity functions determined by Gruppioni et al. (2013) on the basis of Herschel/PACS and SPIRE surveys, and to the bolometric luminosities represented in the AGN bolometric luminosity functions derived by

\footnotetext{
${ }^{20}$ The minimum integration time available for the MRS is $\sim 30 \mathrm{~s}$ (SLOWMode readout time; Ressler et al. 2015). In this paper we consider a minimum exposure time of $0.1 \mathrm{hr}$, because observations with a lower integration time would be very disadvantageous in term of overhead (see Table 6 in Bouchet et al. 2015; see also Gordon et al. 2015) so we expect that it is unlikely they will be planned.
} 

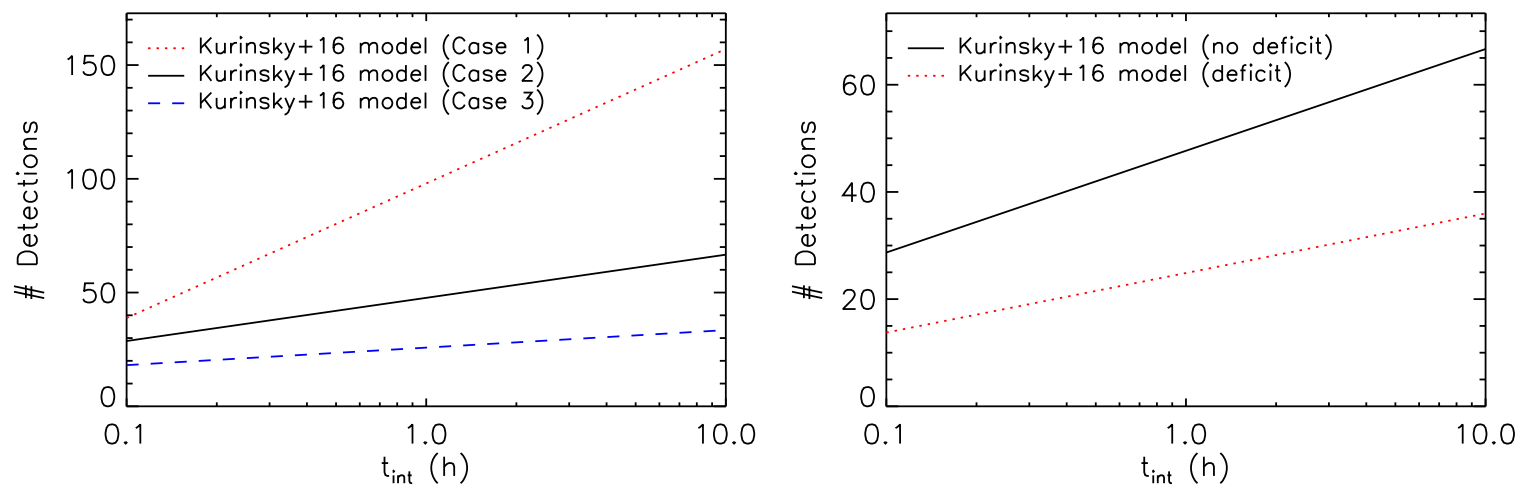

Figure 6. Number of serendipitous $5 \sigma$ line detections per FoV as a function of the integration time. Left: for the Kurinsky et al. (2017) model, comparison between results obtained using the three different assumptions for the low- $L$ end of the IR LF. Right: for the reference model (Kurinsky et al. 2017 model with case 2 low- $L$ end assumption), comparison between detections obtained using the reference correlations between $L_{\mathrm{IR}}$ and $L_{\mathrm{PAH}}$ and those obtained using the assumption of a factor 10 deficit in $L_{\mathrm{PAH}}$ for all the galaxies having $L_{\mathrm{IR}}<10^{9} L_{\odot}$.

Delvecchio et al. (2014) on the basis of the same surveys, respectively.

The exposure times for the PAH and silicate bands have been computed by degrading the resolution in the four MRS channels by a factor of $58,46,45$, and 36 , respectively, to obtain $R \simeq 60$. The suitability of such a resolution for PAH detections has been already demonstrated from the analysis of Spitzer low-resolution spectra (see, e.g., Fiolet et al. 2010; Fleming et al. 2010; O'Dowd et al. 2011). For the fine-structure lines, we considered the full resolution spectra. In other words, we envisage that the full resolution spectra are analyzed first to look for fine-structure lines and then degraded to increase the number of PAH and silicate band detections.

In Figure 4 we see that in a few minutes all the $z=1$ Herschel galaxies are detected in several (at least nine) starforming lines, while at $z=3$ they are detected in four lines, ${ }^{21}$ thus providing spectroscopic redshift measurements for all of them. Figure 5 shows that in the same time, the Herschel sources are detected in at least two pure AGN lines, allowing for the strength of the AGN to be assessed. The implications of these detections are discussed further in Section 5.

\subsection{Serendipitous Detections}

Here we consider the question-for a given exposure time, how many additional (serendipitous) sources should be detected in the same MRS FoV? Figure 6 shows the number of $5 \sigma$ line detections in a single exposure, as a function of the integration time. The left panel of Figure 6 shows the effect of the different assumptions about the faint end of the LFs. On the right, we show how an extreme assumption about the PAH emission of low-luminosity galaxies-simulated galaxies with $L_{\mathrm{IR}}<10^{9} L_{\odot}$ are under-luminous by a factor of 10 in the PAH lines-affects the results. Globally, the number of detections per FoV varies from a few tens (with $0.1 \mathrm{hr}$ integration) to tens/a few hundreds (with $10 \mathrm{hr}$ ). Testing these results by the use of the Cai et al. (2013) model, we found differences within only about a factor of two in the numbers obtained. Even for the shallowest observations, combining a few pointed

\footnotetext{
${ }^{21}$ Note that the Herschel star-forming galaxies are detected in these lines, as well as the Herschel AGNs. As pointed out by Gruppioni et al. (2013), the SF component dominates the total IR luminosity of the Herschel sources, even in the majority of AGNs. At, for example, $z \sim 3$, if we consider the faintest Herschel AGNs (having $L_{\mathrm{IR}} \sim 2 \times 10^{12} L_{\odot}$ ) with a minimum SF component of 50 percent, its SF-only $L_{\mathrm{IR}}$ is higher than the minimum $L_{\mathrm{IR}}$ achieved by the MRS in the PAH 3.3/6.2 $\mu \mathrm{m}, \mathrm{Pa} \alpha$, and [Ar II] $6.98 \mu \mathrm{m}$ lines.
}

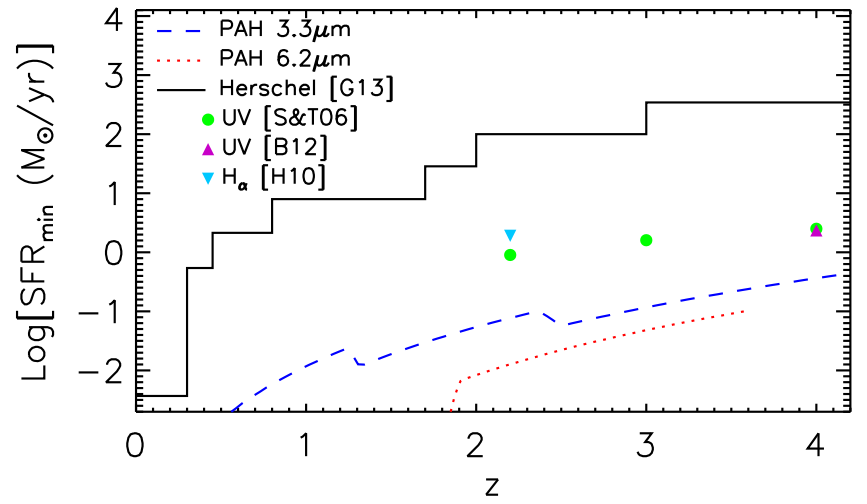

Figure 7. As a function of the redshift, comparison between the minimum SFR achieved by a MRS serendipitous survey with $0.1 \mathrm{hr}$ integration (through spectroscopic detections of PAH 3.3 and $6.2 \mu \mathrm{m}$ lines) and the minimum SFR reached by other IR (SFR corresponding to the minimum luminosities represented in the IR luminosity functions determined by Gruppioni et al. 2013 [G13] on the basis of Herschel/PACS and SPIRE surveys), $\mathrm{H}_{\alpha}$ (Hayes et al. 2010 [H10]), and UV (Sawicki \& Thompson 2006 [S\&T06]; Bouwens et al. 2012 [B12]) measurements. The minimum SFR achieved by the MRS in the two PAH 3.3 and $6.2 \mu \mathrm{m}$ lines was calculated considering the detection limit at $0.1 \mathrm{hr}$ integration, our calibrations between PAH and IR luminosity and the $L_{\mathrm{IR}} / \mathrm{SFR}$ relation derived by Clemens et al. (2013).

observations of Herschel-selected sources would yield statistically significant samples of serendipitous detections. These studies allow us to discriminate between the different scenarios (i.e., they give us insight into the number density and properties of low- $L$ galaxies).

Most ( $>83$ percent in all cases) of the detected lines are PAHs, as a direct consequence of their brightness, of the much greater abundance of star-forming galaxies (compared to AGN) and of the degraded spectral resolution. The fraction of AGN detections (i.e., detections of pure AGN lines) is always $<6$ percent. The implications of these detections are discussed further in Section 5.

\section{Discussion}

\subsection{Implications for the Study of the Galaxy-AGN Co-Evolution}

The study of galaxy-AGN (co-)evolution ultimately requires two different yet complementary approaches. First, we need to understand the detailed physical conditions in galaxies while they are actively star forming and growing their central black 


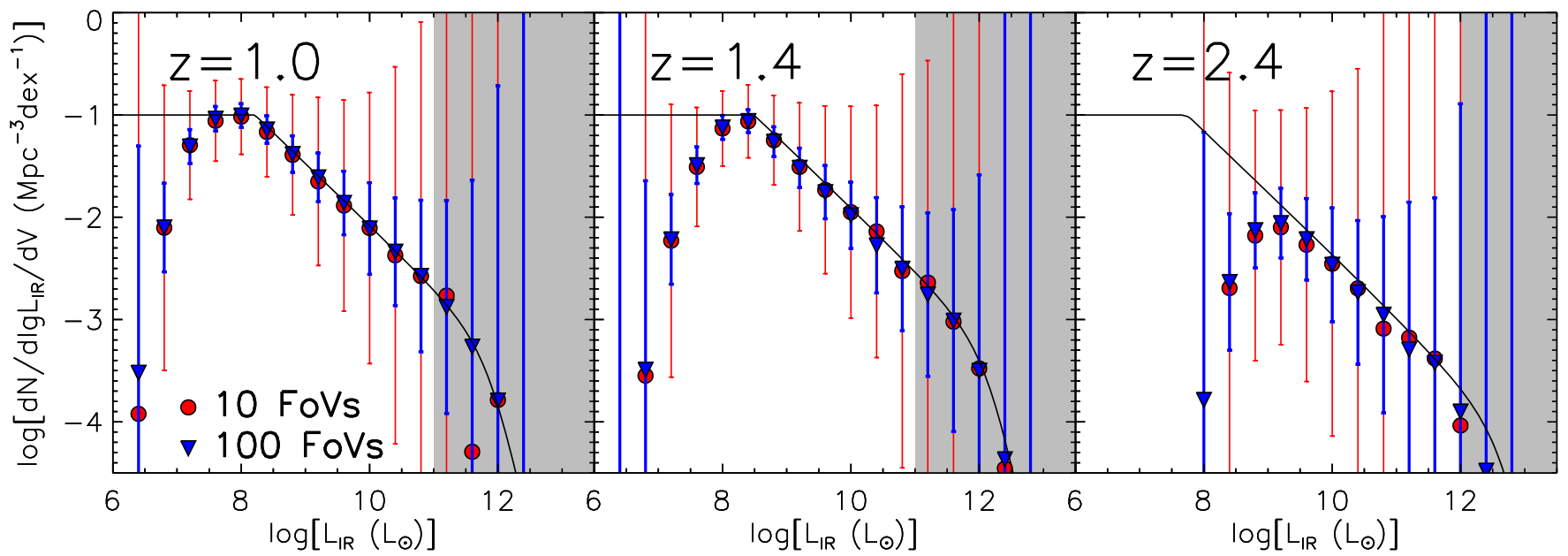

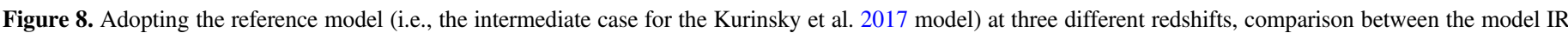

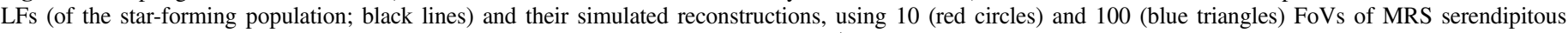
detections with $0.1 \mathrm{hr}$ integration. The shaded regions indicate the regime of Herschel/PACS and SPIRE-detected sources (based on Gruppioni et al. 2013).

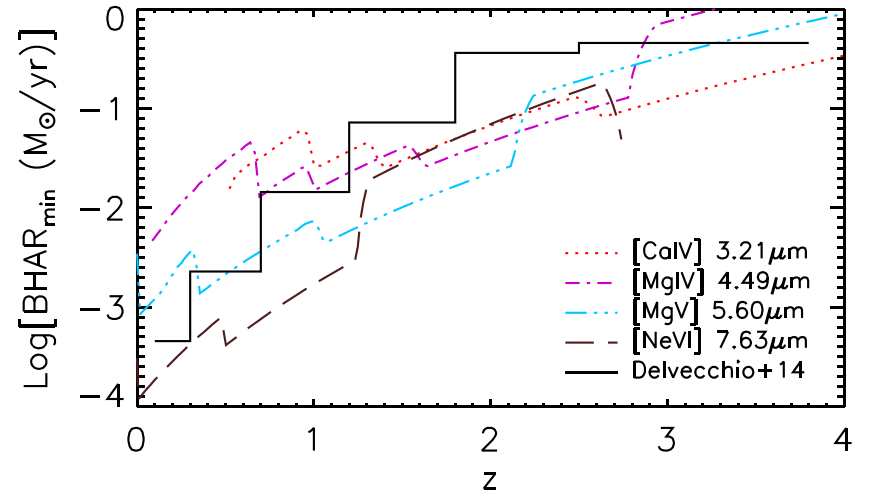

Figure 9. Comparison between the minimum BHAR achieved by a MRS serendipitous survey with $0.1 \mathrm{hr}$ integration (through spectroscopic detections of several AGN lines) and the BHAR corresponding to the minimum bolometric luminosities represented in the AGN bolometric luminosity functions determined by Delvecchio et al. (2014) on the basis of Herschel/ PACS and SPIRE surveys, as a function of the redshift. The minimum BHAR achieved by the MRS in these four different AGN lines was calculated considering the detection limit at $0.1 \mathrm{hr}$ integration, our calibrations between line and AGN bolometric luminosity, and the $L_{\mathrm{bol}} / \mathrm{BHAR}$ relation derived by Chen et al. (2013).

holes. Then we need better constraints on the cosmic history of SF and black hole accretion.

\subsubsection{The Role of AGN in Herschel-selected Galaxies}

Figures $4-5$ show that in $\sim 0.1 \mathrm{hr}$ all Herschel-selected sources will be detected by MRS-pointed observations in multiple lines, thus providing spectroscopic redshift measurements for all of them. The relative roles of AGN and SF in powering all Herschel-selected galaxies can be assessed across this full redshift range by considering the $\mathrm{PAH}$ equivalent widths (e.g., Armus et al. 2007; Sajina et al. 2007; Dasyra et al. 2009). For wide redshift ranges, these can be crosscalibrated using ratios of AGN to SF powered fine-structure lines, which provide more accurate estimates than PAH EWs. Among the best AGN diagnostic lines for the MRS is [Ne VI] $7.65 \mu \mathrm{m}$, a strong AGN line (see Spinoglio \& Malkan 1992) detectable over a broad redshift range from $z=0$ to $z \sim 2.8$. Within this redshift range, detecting the [Ne VI] $7.65 \mu \mathrm{m}$ line in Herschel-selected sources requires only $\sim 0.1 \mathrm{hr}$. A commonly used SF diagnostic line is [Ne II] $12.81 \mu \mathrm{m}$, which can be detected in any Herschel source by the MRS in the range $0<z \lesssim 1.2$ in $\sim 0.1 \mathrm{hr}$. Beyond this redshift, up to $z \sim 3.1$, and with the same integration time, we can use the [Ar II] $6.98 \mu \mathrm{m}$ SF line.

In $\lesssim 1 \mathrm{hr}$, the [Ne III] $15.55 \mu \mathrm{m} /[\mathrm{Ne}$ II] $12.81 \mu \mathrm{m}$ versus [S IV] $10.49 \mu \mathrm{m} /[\mathrm{Ne}$ II] $12.81 \mu \mathrm{m}$ diagnostic plot can be applied to all Herschel galaxies with $z \lesssim 0.9$. This plot can be used to model the age, metallicity, and ionization parameter of star-forming galaxies (see, e.g., Inami et al. 2013). These line ratios can also be used (as in Inami et al. 2013) to constrain photoionization models, for example, using the MAPPINGS III photoionization code (Binette et al. 1985; Sutherland \& Dopita 1993; Groves et al. 2004) in combination with the Starburst99 stellar population synthesis code (Leitherer et al. 1999; Vázquez \& Leitherer 2005).

\subsubsection{The Cosmic SF and Black Hole Accretion Rate Histories and the Low-L End of the IR Luminosity Function}

Serendipitously detected galaxies could be used to trace the SFR history. Figure 7 shows the minimum SFR (calculated using our line/IR luminosity relations and the $L_{\mathrm{IR}} / \mathrm{SFR}$ relation by Clemens et al. 2013) of the sources detectable by the MRS (for $0.1 \mathrm{hr}$, which is the minimum exposure time needed for the detection of Herschel sources). Also shown, for comparison, are the minimum SFR detected in existing Herschel PACS +SPIRE surveys (Gruppioni et al. 2013). The improvement over Herschel is impressive, about three orders of magnitude. Comparison with the lowest SFR achieved by the deepest $\mathrm{H}_{\alpha}$ (Hayes et al. 2010) and UV (Sawicki \& Thompson 2006; Bouwens et al. 2012) surveys at high $z$ also shows a significant improvement achievable through the MRS serendipitous detections. Using such survey data we will sample SFRs well below those of the most efficient star formers at the peak of the cosmic SF activity, at $z$ between 2 and 3, estimated to be $\simeq 100 M_{\odot} \mathrm{yr}^{-1}$ (Genzel et al. 2006; Cai et al. 2013).

For a good reconstruction of the IR LFs, and therefore of the SFR history, through serendipitous spectroscopic detections in $0.1 \mathrm{hr}$ integration, we find that $\sim 100$ FoVs will be necessary. For the reference model (i.e., the intermediate case of the Kurinsky et al. 2017 model), Figure 8 shows IR LFs (of the star-forming population) reconstructed at three different 


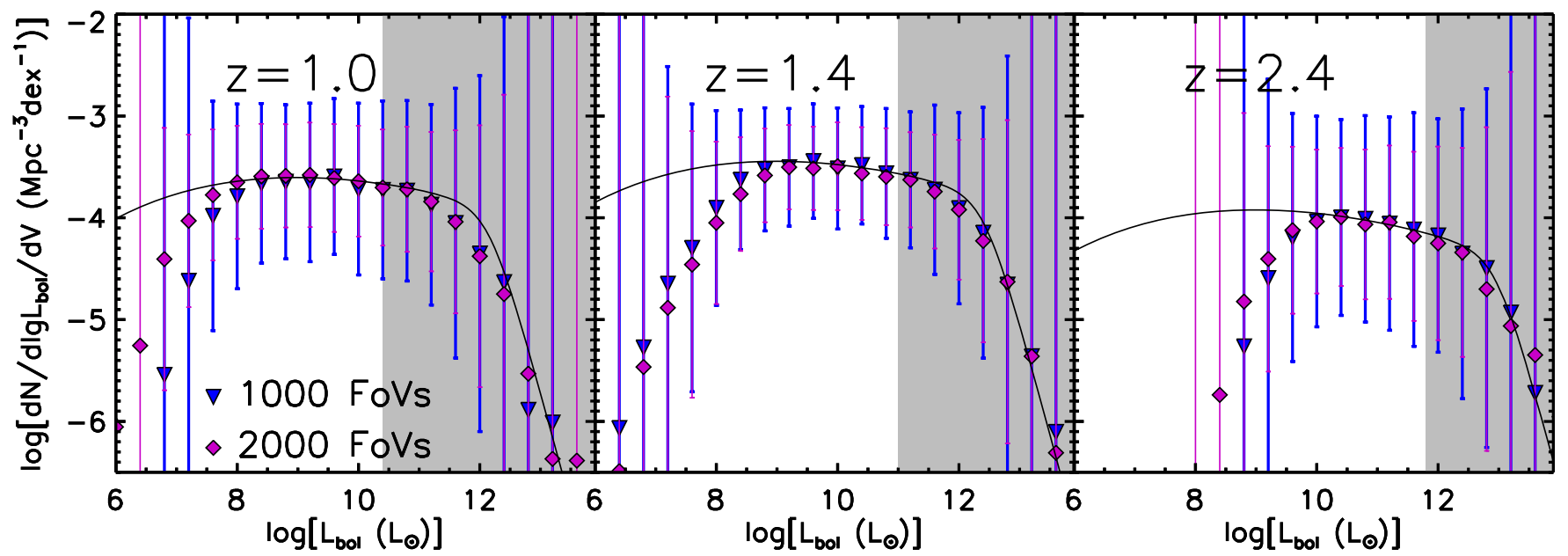

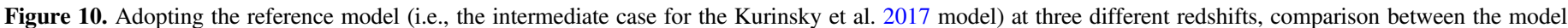

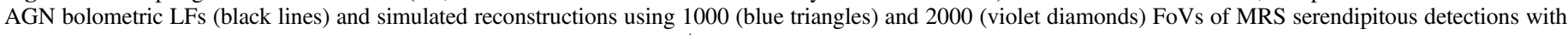
$0.1 \mathrm{hr}$ integration. The shaded regions indicate the regime of Herschel/PACS and SPIRE-detected sources (based on Delvecchio et al. 2014).

redshifts, using serendipitous sources collected from 10 and $100 \mathrm{FoVs}$ and detected in at least two spectral lines.

The LFs in the different bins in redshift and IR luminosity (adopting bin sizes $\Delta z=0.2$ and $\Delta \log L=0.4$ ) were derived using the $1 / V_{\max }$ method (Schmidt 1968):

$$
\frac{d N\left(L_{j}, z_{k}\right)}{d \log L}=\frac{1}{\Delta \log L} \sum_{i=1}^{N_{j}} \frac{1}{V_{\max , i}\left(z_{k}\right)}
$$

where $z_{k}$ is the bin center and the sum is over all the $N_{j}$ sources with luminosity in the range $\left[\log L_{j}-\Delta \log L / 2, \log L_{j}+\right.$ $\Delta \log L / 2]$ within the redshift bin. The quantity $V_{\max , i}$ is the comoving volume, within the solid angle of the survey, enclosed between the lower $\left(z_{\min }\right)$ and the upper $\left(z_{\max }\right)$ limit of the bin. The mean $d N\left(L_{j}, z_{k}\right) / d \log L$ obtained from the simulations was adopted as the maximum likelihood value. The dispersions around the mean $d N\left(L_{j}, z_{k}\right) / d \log L$ were taken as estimates of the Poisson errors, calculated as: $\sigma_{j, k}=\left[\sum_{i=1}^{N_{j}}\left(\frac{1}{V_{\max , i}}\left(z_{k}\right)\right)^{2}\right]^{1 / 2}$.

Note that the contribution of very low- $L$ sources (placed in the regions of our three different model LF low- $L$ end) to the star formation rate density (SFRD) is minor (see Madau \& Dickinson 2014). However these serendipitous surveys will be able to explore levels of SFR never achieved before. They will provide an impressive improvement in the reconstruction of the faint end of the PAH line LFs, and therefore of the IR LFs and of the SFR functions.

Considering the simulated reconstructions of the LFs described above and using the first seven bins in redshift (until $z=1.4$; i.e., where the incompleteness does not affect significantly the measurement, ${ }^{22}$ see Figure 8), we obtain a value for the slope of $\sim 0.1 \pm 0.3$ with 100 FoVs. With a smaller number of FoVs, the uncertainty on this measurement would be too high (e.g., we obtain a slope $\sim 0.2 \pm 1.9$ with 10

\footnotetext{
${ }^{22}$ This incompleteness effect is strictly correlated to the dispersion in the $L_{\text {line }}$ $L_{\mathrm{IR}}$ relationships (i.e., to the fact that, close to the spectroscopic detection limit, only a fraction of the galaxies with a certain $L_{\mathrm{IR}}$ has a $L_{\text {line }}$ greater than such detection limit).
}

FoVs). But about $100 \mathrm{FoVs}$ will provide a tight estimation of the slope of the low- $L$ end.

In Figure 9 we compare, as a function of redshift, the minimum black hole accretion rates (BHARs; calculated using our line/bolometric luminosity relations and the $L_{\mathrm{bol}} / \mathrm{BHAR}$ relation by Chen et al. 2013) of the sources detectable by the MRS (again in $0.1 \mathrm{hr}$ integration) with those associated with the minimum bolometric luminosities represented in the AGN bolometric luminosity functions determined by Delvecchio et al. (2014) on the basis of Herschel/PACS and SPIRE surveys. We find an improvement over Herschel of about one order of magnitude.

Despite this improvement in sensitivity, because of the relative rarity of AGNs, a large number of FoVs is necessary (i.e., $\gtrsim 1000$, according to our simulations) for a good reconstruction of the AGN bolometric LFs, and therefore of the BHAR functions, again with $0.1 \mathrm{hr}$ integration. Figure 10 shows AGN bolometric LFs reconstructed at three different redshifts using serendipitous sources collected from 1000 and 2000 FoVs and detected in at least two spectral lines.

\subsection{Implications for the Study of High-z Low-L Galaxies}

Figure 11 shows the luminosity distributions of serendipitously detected galaxies detectable in at least two lines-per FoV and for an integration time of $0.1 \mathrm{hr}$. We compare this distribution for the three different assumptions about the lowluminosity end of the IR LF and including or not a PAH deficit. We can see that in all cases the peak of the detected sources is located at very low luminosities $\left(\lesssim 10^{9} L_{\odot}\right)$. Figure 12 shows how the $\log \left(L_{\mathrm{IR}}\right)-z$ plane distribution of the detected (in at least two lines) sources varies with the PAH deficit assumption. The peak of the detectable galaxies is located at IR luminosities $\lesssim 10^{9} L_{\odot}$ and at redshifts $\sim 1-2$. Using the Cai et al. (2013) model, we obtained consistent results. Therefore the considered scenarios all suggest that, for the first time, MIRI MRS will be able to detect in a few minutes extremely faint galaxies, up to high redshifts, and with good statistics.

Here we consider sources detectable in at least two lines, because robust redshift measurements will be provided, enabling reconstruction of the low- $L$ end of the IR luminosity function. 

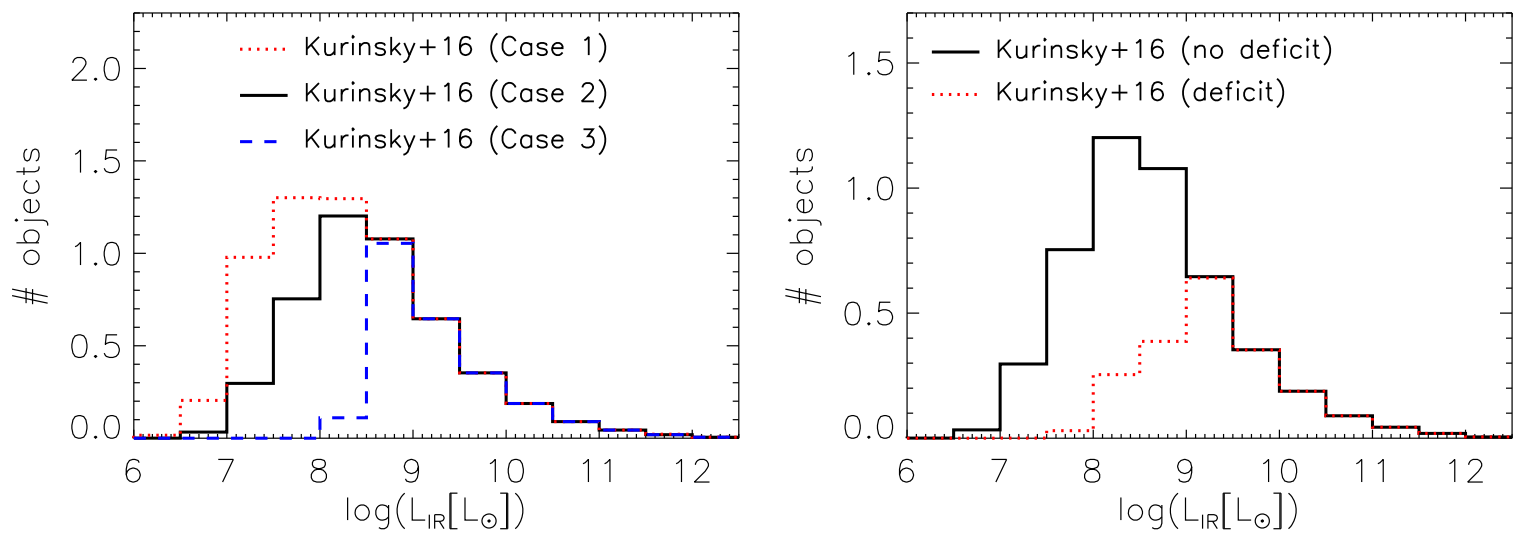

Figure 11. Predicted IR luminosity distributions of galaxies detectable in at least two lines (with $0.1 \mathrm{hr}$ integration). On the left: for the Kurinsky et al. (2017) model, comparison between the results obtained using the three different assumptions for the low- $L$ end of the IR LFs. On the right: for the reference model (Kurinsky et al. 2017 with case 2 low- $L$ end assumption), comparison between the predictions obtained using the reference correlations between $L_{\mathrm{IR}}$ and $L_{\mathrm{PAH}}$ and those obtained using the assumption of a factor 10 deficit in $L_{\mathrm{PAH}}$ for all the galaxies with $L_{\mathrm{IR}}<10^{9} L_{\odot}$.
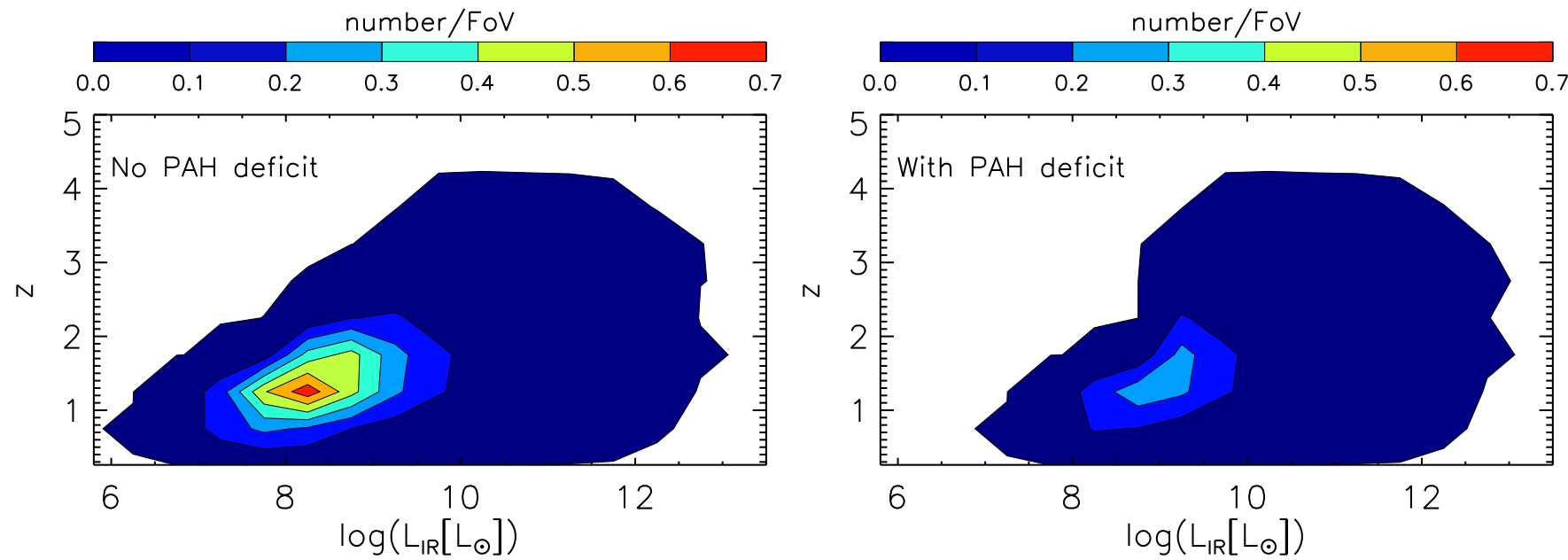

Figure 12. Two-dimensional density function on the $\log \left(L_{\mathrm{IR}}\right)-z$ plane of the predicted sources detectable in at least two lines by a MIRI MRS serendipitous survey covering the area of the FoV in $0.1 \mathrm{hr}$ integration. On the left: predictions for the reference model (Kurinsky et al. 2017 with case 2 low- $L$ end assumption) and the reference correlations between $L_{\mathrm{IR}}$ and $L_{\mathrm{PAH}}$. On the right: predictions for the reference model and the assumption of a factor 10 deficit in $L_{\mathrm{PAH}}$ (for all the galaxies having $L_{\mathrm{IR}}<10^{9} L_{\odot}$ ). The different colors correspond to the number of detected sources (see the color bar on the top).

However, we verified that when considering sources detectable in at least one or three lines, the peaks of the redshift and luminosity distributions are generally located in the same bins as the two-line case. The only difference is in the number of sources detected, which increases by $\sim 40-50$ percent in the oneline case and decreases by $\sim 10-20$ percent in the three-line case.

These galaxies will be detected in PAH lines. As mentioned in Section 1, the relationship between line and continuum emission in this luminosity regime is highly uncertain, and probably only a minor fraction of the IR luminosity is due to SF (see Section 3.1). However, follow-up observations of these sources would permit us to study the evolution of their physical properties and derive diagnostics and indicators that are currently calibrated only for their high- $L$ counterparts.

For example, observing these low- $L$ serendipitous sources with the JWST Near-Infrared Spectrograph ${ }^{23}$ (NIRSpec; Posselt et al. 2004; Ferruit et al. 2012) in medium-resolution spectroscopy would allow us to detect them in both $\mathrm{H}_{\alpha}$ and

\footnotetext{
$\overline{23}$ It will provide integral-field spectroscopy in the $1-5 \mu \mathrm{m}$ wavelength range in medium resolution.
}

$\mathrm{H}_{\beta}$ lines (for galaxies at $z \gtrsim 1$ ). Combining NIRSpec $\mathrm{H}_{\alpha} / \mathrm{H}_{\beta}$ measurements with MRS PAH ones for a sample of serendipitous $(z \gtrsim 1)$ low- $L$ galaxies will allow us to calibrate the PAH luminosity as SFR indicator in this regime, through the extinction-corrected $\mathrm{H}_{\alpha}$ luminosity (corrected from the Balmer decrement; ratio of $\mathrm{H}_{\alpha} / \mathrm{H}_{\beta}$ ). A similar approach to the method is presented in Shipley et al. (2016) for high- $L$ starforming galaxies.

\section{Conclusions}

In this paper we build upon earlier works in Bonato et al. (2014a, 2014b, 2015) that include relations for line to total IR luminosity for a large number of SF and AGN mid-IR spectral lines. With these, we make specific predictions for the detectability of said lines in different redshift and IR luminosity regimes. We find that only a few minutes' integration of MRSpointed observations of Herschel-selected sources are sufficient to obtain spectroscopic redshifts for all of them and to investigate the role of $\mathrm{SF}$ and $\mathrm{AGN}$ therein through a combination of PAH equivalent widths and fine-structure line ratios. 
We use the recent model for the evolution of the IR LF from Kurinsky et al. (2017), coupled with three different assumptions for the unknown low- $L$ slope of the luminosity function, and accounting for the PAH deficit in low- $L$, low-metallicity galaxies to investigate the likely number serendipitous galaxy detections in the same pointed MRS observations described above. We find that each $0.1 \mathrm{hr}$ pointed MRS observation can result in tens of serendipitous detections. Most ( $>83$ percent) are PAHs, while AGN detections are only $<6$ percent of the total.

Such serendipitous surveys will allow us, for the first time and for free, to detect very low-luminosity galaxies up to high redshifts, and with good statistics. The bulk of these sources have $\sim 10^{8-9} L_{\odot}$ and $z \sim 1-2$. This is a completely unexplored regime. Thus serendipitous MIRI surveys will allow us to study the properties of these intermediate redshift dwarf galaxies and to test galaxy evolution models, in particular the faint end of the IR LFs. In fact, serendipitous detections collected from about 100 FoVs (with $0.1 \mathrm{hr}$ integration) will be sufficient for a tight estimation of the slope of the low- $L$ end.

These serendipitous observations will be able to achieve unexplored levels of SFR and BHAR. Even with short integration times of a few minutes, the improvement over Herschel is impressive: about three orders of magnitude in SFR and one order in BHAR. This inclusion of lower luminosity sources will allow us to reconstruct the PAH line luminosity functions, continuum luminosity functions, and SFR functions (with $\sim 100 \mathrm{FoVs}$ in $0.1 \mathrm{hr}$ integration) much more accurately than currently possible.

We thank Prof. George Rieke for helpful discussions. We are grateful to the anonymous referee for many constructive comments that helped us improve this paper. M.B. is supported by NASA-ADAP13-0054. A.S. and J.M. acknowledge support through NSF AAG\#1313206. A.P. acknowledges support from NSF AAG \#1312418. M.N. has received funding from the European Unions Horizon 2020 research and innovation programme under the Marie Sklodowska-Curie grant agreement No 707601.

\section{References}

Armus, L., Charmandaris, V., Bernard-Salas, J., et al. 2007, ApJ, 656, 148 Bernard-Salas, J., Spoon, H. W. W., Charmandaris, V., et al. 2009, ApJS, 184,230

Binette, L., Dopita, M. A., \& Tuohy, I. R. 1985, ApJ, 297, 476

Bonato, M., Negrello, M., Cai, Z.-Y., et al. 2014a, MNRAS, 438, 2547

Bonato, M., Negrello, M., Cai, Z.-Y., et al. 2014b, MNRAS, 444, 3446

Bonato, M., Negrello, M., Cai, Z.-Y., et al. 2015, MNRAS, 452, 356

Bouchet, P., García-Marín, M., Lagage, P.-O., et al. 2015, PASP, 127, 612

Bourne, N., Dunne, L., Maddox, S. J., et al. 2016, arXiv:1606.09254

Bouwens, R. J., Illingworth, G. D., Oesch, P. A., et al. 2012, ApJ, 754, 83

Bruce, V. A., Dunlop, J. S., Cirasuolo, M., et al. 2012, MNRAS, 427, 1666

Burgarella, D., Buat, V., Gruppioni, C., et al. 2013, A\&A, 554, A70

Cai, Z.-Y., Lapi, A., Xia, J.-Q., et al. 2013, ApJ, 768, 21

Caputi, K. I., Lagache, G., Yan, L., et al. 2007, ApJ, 660, 97

Chen, C.-T. J., Hickox, R. C., Alberts, S., et al. 2013, ApJ, 773, 3

Clemens, M. S., Negrello, M., De Zotti, G., et al. 2013, MNRAS, 433, 695

Contursi, A., Lequeux, J., Cesarsky, D., et al. 2000, A\&A, 362, 310

Contursi, A., Lequeux, J., Hanus, M., et al. 1998, A\&A, 336, 662

Dasyra, K. M., Ho, L. C., Netzer, H., et al. 2011, ApJ, 740, 94

Dasyra, K. M., Yan, L., Helou, G., et al. 2009, ApJ, 701, 1123

Delvecchio, I., Gruppioni, C., Pozzi, F., et al. 2014, MNRAS, 439, 2736

Desai, V., Armus, L., Spoon, H. W. W., et al. 2007, ApJ, 669, 810

Díaz-Santos, T., Charmandaris, V., Armus, L., et al. 2010, ApJ, 723, 993

Dopita, M. A., Fischera, J., Sutherland, R. S., et al. 2006, ApJS, 167, 177

Driver, S. P., Robotham, A. S. G., Kelvin, L., et al. 2012, MNRAS, 427, 3244
Eales, S., Dunne, L., Clements, D., et al. 2010, PASP, 122, 499 Elbaz, D., Dickinson, M., Hwang, H. S., et al. 2011, A\&A, 533, A119 Engelbracht, C. W., Gordon, K. D., Rieke, G. H., et al. 2005, ApJL, 628, L29 Engelbracht, C. W., Rieke, G. H., Gordon, K. D., et al. 2008, ApJ, 678, 804 Farrah, D., Bernard-Salas, J., Spoon, H. W. W., et al. 2007, ApJ, 667, 149 Ferrarese, L., \& Ford, H. 2005, SSRv, 116, 523

Ferruit, P., Bagnasco, G., Barho, R., et al. 2012, Proc. SPIE, 8442, 844220 Finkelstein, S. L., Dunlop, J., Le Fevre, O., \& Wilkins, S. 2015, arXiv:1512. 04530

Fiolet, N., Omont, A., Lagache, G., et al. 2010, A\&A, 524, A33

Fisher, D. B., Bolatto, A. D., Herrera-Camus, R., et al. 2014, Natur, 505, 186 Fleming, B., France, K., Lupu, R. E., \& McCandliss, S. R. 2010, ApJ, 725, 159 Förster Schreiber, N. M., Roussel, H., Sauvage, M., \& Charmandaris, V. 2004, A\&A, 419, 501

Galametz, M., Madden, S., Galliano, F., et al. 2009, A\&A, 508, 645

Gallagher, J. S., III, Hunter, D. A., Gillett, F. C., \& Rice, W. L. 1991, ApJ, 371,142

Galliano, F., Madden, S. C., Jones, A. P., et al. 2003, A\&A, 407, 159

Gardner, J. P., Mather, J. C., Clampin, M., et al. 2006, SSRv, 123, 485

Genzel, R., Lutz, D., Sturm, E., et al. 1998, ApJ, 498, 579

Genzel, R., Tacconi, L. J., Eisenhauer, F., et al. 2006, Natur, 442, 786

Glasse, A., Rieke, G. H., Bauwens, E., et al. 2015, PASP, 127, 686

Gordon, K. D., Chen, C. H., Anderson, R. E., et al. 2015, PASP, 127, 696

Graham, A. W., \& Scott, N. 2013, ApJ, 764, 151

Groves, B. A., Dopita, M. A., \& Sutherland, R. S. 2004, ApJS, 153, 75

Gruppioni, C., Pozzi, F., Rodighiero, G., et al. 2013, MNRAS, 432, 23

Hayes, M., Schaerer, D., \& Östlin, G. 2010, A\&A, 509, L5

Ho, L. C., \& Keto, E. 2007, ApJ, 658, 314

Hopkins, P. F., Hernquist, L., Cox, T. J., et al. 2006, ApJS, 163, 1

Hopkins, P. F., Hernquist, L., Cox, T. J., \& Kereš, D. 2008, ApJS, 175, 356

Houck, J. R., Charmandaris, V., Brandl, B. R., et al. 2004a, ApJS, 154, 211

Houck, J. R., Roellig, T. L., Van Cleve, J., et al. 2004b, Proc. SPIE, 5487, 62 Hummer, D. G., \& Storey, P. J. 1987, MNRAS, 224, 801

Hunter, D. A., Kaufman, M., Hollenbach, D. J., et al. 2001, ApJ, 553, 121

Imanishi, M., Nakagawa, T., Ohyama, Y., et al. 2008, PASJ, 60, S489

Imanishi, M., Nakagawa, T., Shirahata, M., Ohyama, Y., \& Onaka, T. 2010, ApJ, 721, 1233

Inami, H., Armus, L., Charmandaris, V., et al. 2013, ApJ, 777, 156

Israel, F. P., Bontekoe, T. R., \& Kester, D. J. M. 1996, A\&A, 308, 723

Jackson, D. C., Cannon, J. M., Skillman, E. D., et al. 2006, ApJ, 646, 192

Jahnke, K., \& Macciò, A. V. 2011, ApJ, 734, 92

Kendrew, S., Scheithauer, S., Bouchet, P., et al. 2015, PASP, 127, 623

Kennicutt, R. C., Jr. 1998, ARA\&A, 36, 189

Kim, J. H., Im, M., Lee, H. M., et al. 2012, ApJ, 760, 120

Kirkpatrick, A., Pope, A., Alexander, D. M., et al. 2012, ApJ, 759, 139

Kirkpatrick, A., Pope, A., Sajina, A., et al. 2015, ApJ, 814, 9

Kormendy, J., \& Ho, L. C. 2013, ARA\&A, 51, 511

Kurczynski, P., Gawiser, E., Acquaviva, V., et al. 2016, ApJL, 820, L1

Kurinsky, N., Sajina, A., Bonato, M., et al. 2017, ApJ, submitted

Lapi, A., González-Nuevo, J., Fan, L., et al. 2011, ApJ, 742, 24

Lee, J. C., Hwang, H. S., Lee, M. G., Kim, M., \& Lee, J. H. 2012, ApJ, 756, 95

Le Floc'h, E., Papovich, C., Dole, H., et al. 2005, ApJ, 632, 169

Leitherer, C., Schaerer, D., Goldader, J. D., et al. 1999, ApJS, 123, 3

Lutz, D. 2014, ARA\&A, 52, 373

Lutz, D., Veilleux, S., \& Genzel, R. 1999, ApJL, 517, L13

Madau, P., \& Dickinson, M. 2014, ARA\&A, 52, 415

Madden, S. C., Galliano, F., Jones, A. P., \& Sauvage, M. 2006, A\&A, 446, 877 Madden, S. C., Rémy-Ruyer, A., Galametz, M., et al. 2013, PASP, 125, 600

Magnelli, B., Elbaz, D., Chary, R. R., et al. 2009, A\&A, 496, 57

Magnelli, B., Elbaz, D., Chary, R. R., et al. 2011, A\&A, 528, A35

Magnelli, B., Popesso, P., Berta, S., et al. 2013, A\&A, 553, A132

Merloni, A., \& Heinz, S. 2008, MNRAS, 388, 1011

Negrello, M., Clemens, M., Gonzalez-Nuevo, J., et al. 2013, MNRAS, 429, 1309

O’Dowd, M. J., Schiminovich, D., Johnson, B. D., et al. 2009, ApJ, 705, 885

O’Dowd, M. J., Schiminovich, D., Johnson, B. D., et al. 2011, ApJ, 741, 79

O’Halloran, B., Satyapal, S., \& Dudik, R. P. 2006, ApJ, 641, 795

Oliver, S. J., Bock, J., Altieri, B., et al. 2012, MNRAS, 424, 1614

Peeters, E., Spoon, H. W. W., \& Tielens, A. G. G. M. 2004, ApJ, 613, 986

Peng, C. Y. 2007, ApJ, 671, 1098

Peng, Y.-j., Lilly, S. J., Kovač, K., et al. 2010, ApJ, 721, 193

Planck Collaboration 2015, arXiv:1502.01589

Pope, A., Chary, R.-R., Alexander, D. M., et al. 2008, ApJ, 675, 1171

Posselt, W., Holota, W., Kulinyak, E., et al. 2004, Proc. SPIE, 5487, 688

Reach, W. T., Boulanger, F., Contursi, A., \& Lequeux, J. 2000, A\&A, 361, 895

Rémy-Ruyer, A., Madden, S. C., Galliano, F., et al. 2013, A\&A, 557, A95 
Ressler, M. E., Sukhatme, K. G., Franklin, B. R., et al. 2015, PASP, 127, 675

Rieke, G. H., Alonso-Herrero, A., Weiner, B. J., et al. 2009, ApJ, 692, 556

Rieke, G. H., Wright, G. S., Böker, T., et al. 2015, PASP, 127, 584

Rodighiero, G., Renzini, A., Daddi, E., et al. 2014, MNRAS, 443, 19

Rodighiero, G., Vaccari, M., Franceschini, A., et al. 2010, A\&A, 515, A8

Rodríguez-Ardila, A., \& Viegas, S. M. 2003, MNRAS, 340, L33

Rosenberg, J. L., Wu, Y., Le Floc'h, E., et al. 2008, ApJ, 674, 814

Roussel, H., Sauvage, M., Vigroux, L., \& Bosma, A. 2001, A\&A, 372, 427

Sajina, A., Spoon, H., Yan, L., et al. 2009, ApJ, 703, 270

Sajina, A., Yan, L., Armus, L., et al. 2007, ApJ, 664, 713

Sajina, A., Yan, L., Fadda, D., Dasyra, K., \& Huynh, M. 2012, ApJ, 757, 13

Sajina, A., Yan, L., Lutz, D., et al. 2008, ApJ, 683, 659

Salmon, B., Papovich, C., Finkelstein, S. L., et al. 2015, ApJ, 799, 183

Sanders, D. B., Mazzarella, J. M., Kim, D.-C., Surace, J. A., \& Soifer, B. T. 2003, AJ, 126, 1607

Sawicki, M., \& Thompson, D. 2006, ApJ, 642, 653

Schmidt, M. 1968, ApJ, 151, 393

Shen, S., Mo, H. J., White, S. D. M., et al. 2003, MNRAS, 343, 978

Shipley, H. V., Papovich, C., Rieke, G. H., et al. 2013, ApJ, 769, 75

Shipley, H. V., Papovich, C., Rieke, G. H., Brown, M. J. I., \& Moustakas, J. 2016, ApJ, 818, 60

Smith, J. D. T., Draine, B. T., Dale, D. A., et al. 2007, ApJ, 656, 770
Spinoglio, L., \& Malkan, M. A. 1992, ApJ, 399, 504

Sturm, E., Lutz, D., Tran, D., et al. 2000, A\&A, 358, 481

Sturm, E., Lutz, D., Verma, A., et al. 2002, A\&A, 393, 821

Sutherland, R. S., \& Dopita, M. A. 1993, ApJS, 88, 253

Tommasin, S., Spinoglio, L., Malkan, M. A., et al. 2008, ApJ, 676, 836

Tommasin, S., Spinoglio, L., Malkan, M. A., \& Fazio, G. 2010, ApJ, 709, 1257

Tremonti, C. A., Heckman, T. M., Kauffmann, G., et al. 2004, ApJ, 613, 898

Valiante, E., Smith, M. W. L., Eales, S., et al. 2016, arXiv:1606.09615

Vázquez, G. A., \& Leitherer, C. 2005, ApJ, 621, 695

Veilleux, S., Rupke, D. S. N., Kim, D.-C., et al. 2009, ApJS, 182, 628

Vermeij, R., Peeters, E., Tielens, A. G. G. M., \& van der Hulst, J. M. 2002, A\&A, 382, 1042

Wells, M., Pel, J.-W., Glasse, A., et al. 2015, PASP, 127, 646

Windhorst, R. A., Mather, J., Clampin, M., et al. 2009, in Astro2010: The Astronomy and Astrophysics Decadal Survey, Galaxies Across Cosmic Time with JWST, Science White Papers, No. 317

Wu, Y., Charmandaris, V., Hao, L., et al. 2006, ApJ, 639, 157

Xu, C. K., Cao, C., Lu, N., et al. 2014, ApJ, 787, 48

Yamada, R., Oyabu, S., Kaneda, H., et al. 2013, PASJ, 65 arXiv:1307.6356

Yan, L., Sajina, A., Fadda, D., et al. 2007, ApJ, 658, 778

Yuan, T.-T., Kewley, L. J., \& Sanders, D. B. 2010, ApJ, 709, 884

Zahid, H. J., Geller, M. J., Kewley, L. J., et al. 2013, ApJL, 771, L19 